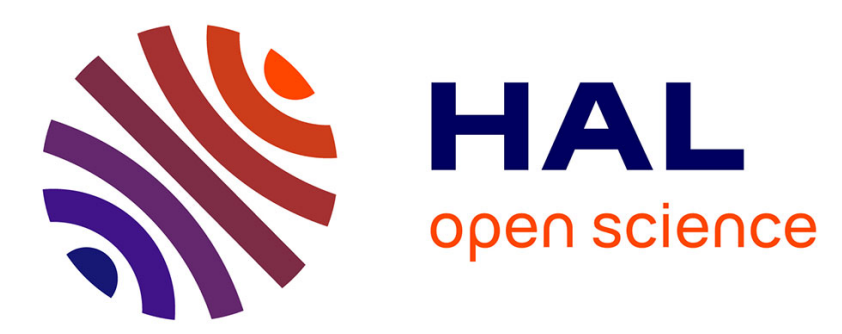

\title{
Edge detection: a review of dissimilarity evaluations and a proposed normalized measure
}

Baptiste Magnier

\section{To cite this version:}

Baptiste Magnier. Edge detection: a review of dissimilarity evaluations and a proposed normalized measure: review. Multimedia Tools and Applications, 2018, 77 (8), pp.9489-9533. 10.1007/s11042017-5127-6 . hal-01940231

\section{HAL Id: hal-01940231 \\ https://hal.science/hal-01940231}

Submitted on 30 Nov 2018

HAL is a multi-disciplinary open access archive for the deposit and dissemination of scientific research documents, whether they are published or not. The documents may come from teaching and research institutions in France or abroad, or from public or private research centers.
L'archive ouverte pluridisciplinaire HAL, est destinée au dépôt et à la diffusion de documents scientifiques de niveau recherche, publiés ou non, émanant des établissements d'enseignement et de recherche français ou étrangers, des laboratoires publics ou privés. 


\title{
Edge Detection: A Review of Dissimilarity Evaluations and a Proposed Normalized Measure
}

\author{
Baptiste Magnier
}

\begin{abstract}
In digital images, edges characterize object boundaries, so edge detection remains a crucial stage in numerous applications. To achieve this task, many edge detectors have been designed, producing different results, with various qualities of segmentation. Indeed, optimizing the response obtained by these detectors has become a crucial issue, and effective contour assessment assists performance evaluation. In this paper, several referenced-based boundary detection evaluations are detailed, pointing out their advantages and disadvantages, theoretically and through concrete examples of image edges. Then, a new normalized supervised edge map quality measure is proposed, comparing a ground truth contour image, the candidate contour image and their associated spatial nearness. The effectiveness of the proposed distance measure is demonstrated theoretically and through several experiments, comparing the results with the methods detailed in the state-of-the-art. In summary, compared to other boundary detection assessments, this new method proved to be a more reliable edge map quality measure.
\end{abstract}

Index Terms-Edge detection, supervised evaluation, distance measures.

\section{INTRODUCTION}

In computer science, all systems, especially automated information processing structures, must be evaluated before being developed, principally for industrial applications or medical data. Image processing is no exception to this rule. In image analysis, image segmentation is one of the most critical tasks and represents an essential step in low-level vision. All the methods developed therefore have to be tested and assessed, whether regarding edge detection, point matching, region segmentation or image restoration/enhancement.

Edge detection represents one of the pioneer theoretical works in image processing tasks [38] and remains a key point in many applications. It is extensively used because boundaries include the most important structures in the image [54][44]. Furthermore, edge detection itself could be used to qualify a region segmentation technique [32][34]. In addition, contour extraction remains a very useful preprocessing step in image segmentation, registration, reconstruction, interpretation and tracking [33]. An efficient boundary detection method should create a contour image containing edges at their correct locations with a minimum of misclassified pixels. Edge detection assessment is therefore an essential field of study, but research has still not necessarily gone deep enough regarding contour detection for digital images. Contrary to region segmentation evaluations, which may consider color attributes, contour detection assessments generally use binary images. Thus, an

Ecole des Mines d'Alès, Site de Croupillac, LGI2P, 7. rue Jules Renard, 30100 ALES, France. E-mail: baptiste.magnier@mines-ales.fr

Special thanks to Adam Clark Gimming.

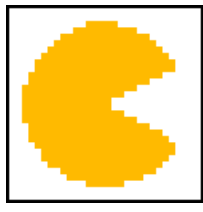

(a) Object

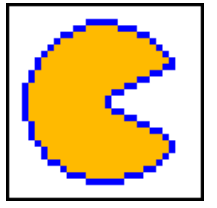

(b) Contour 1

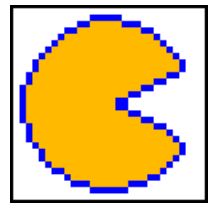

(c) Contour 2

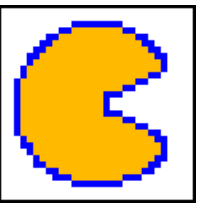

(d) Contour 3
Fig. 1. Several edge chains are available for the same object. The image in (a) is of size $30 \times 30$. Contour points are represented in blue for image (b), (c) and (d). Contour 1 represents the inner edge whereas contour 2 corresponds to the outer boundary. Contour 3 is the result of a step edge detector.

edge detection evaluation leads to a direct assessment of the considered binary image. Moreover, measures of boundary detection evaluation are based on the fact that a reliable edge map should characterize all the relevant structures of the image. It should also create a minimum of spurious pixels or holes (oversights). Therefore, an evaluation can be used to assess and improve an algorithm, or to optimize edge detector parameters [21], for example thresholds in the last stage of the detection.

In edge detection assessment, the measurement process can be classified as using either unsupervised or supervised evaluation criteria. The first class of methods exploits only the input data image and gives a score of coherence that qualifies the result given by the algorithm [21][17][42]. The second class computes a similarity/dissimilarity measure between a segmentation result and a ground truth obtained from synthetic data or an expert judgment [9][22][31]. As edge extraction is performed for image processing tasks and computer vision, similarity measures are important within a broad flied of study, for example in image interpretation, consisting in automatically extracting or recognizing objects in an image, compared to a model. Moreover, the assessment can compute a similarity or a dissimilarity of the shapes attributes between two of binary objects [13][22][37]. Thus, the problem of supervised edge detection assessment amounts to a question of pattern recognition. Nevertheless, the edge position of an object could be interpreted in different ways, as represented in Fig. 1 Indeed, for a vertical step edge, an edge can be located either on the left, or on the right (which corresponds to an inner or an outer contour). Considering a vertical blurred edge (i.e. ramp contour [6]), the true edge is placed in the middle of the blur, but the question of the position remains the same when the size of the blur is even. In the crest lines case [37], true edges are chosen in the middle of the ridge or of the valley when the width of the ridge/valley is equal to a odd number (i.e. at the maxima -top of ridges- or minima -bottom 
of valleys- [36] ). Finally, for a single real or synthetic image, as several contour chains constituting the ground truth depend on several positions of the true pixels, thus the combinations of the boundary pixel placements are numerous, so many chains could be created/chosen. For example, we can create a ground truth boundary map of the image (a) in Fig. 1 with as many combinations as there are pixels in Contour 1,2 and 3. This observation shows that the evaluation must not be binary, but rather illustrates the importance of taking into account the misplaced edge distance regarding the desired contour of the ground true image. For all these reasons, as described in [44], quantitative assessments are generally lacking in proposed edge detection methods.

In image segmentation evaluation, the Structural Similarity Index $(S S I M)$ estimates the visual impact of shifts in an image [57]. This measure is based on the grayscale information concerning the attributes that represent the structure of objects in the scene. SSIM consists of three local comparison functions, namely luminance comparison, contrast comparison, and structure comparison between two signals excluding other remaining errors. Unlike the $P S N R$ (Peak Signal to Noise Ratio) or RMSE (Root-Mean-Square Error), which are measured at the global or the image level, the SSIM is computed locally by moving a $8 \times 8$ window for each pixel. The final score of an entire image corresponds to the mean of all the local scores. Applications of this image quality evaluation include automatically judging the performance of compression or restoration algorithms, concerning grayscale or color images. Even though $S S I M$ can be applied in the case of an edge detection evaluation, in the presence of too many areas without contours, the obtained score is not efficient or useful (in order to judge the quality of edge detection with the $S S I M$, it is necessary to compare with an image having detected edges situated throughout the image areas).

This work focusses on comparisons of supervised edge detection evaluations with respect to binary representation of the boundaries. As introduced above, a supervised evaluation process estimates scores between a ground truth and a candidate edge map (both binary images). These scores could be evaluated counting the number of erroneous pixels, but also through spatial distances of misplaced or undetected contours; this paper outlines the various algorithms presented in the literature. In addition, a new supervised edge map quality measure based on the distances of misplaced pixels is presented and compared to the others; the score obtained by this measure is normalized and can be interpreted for each type of contour. In order to present the effectiveness and efficiency of the proposed method, this new formula is compared with others presented and detailed in the state-of-theart. Thus, several comparisons of edge detection assessments are carried out for different perturbations such as: addition of false positive points, creation of false negative points, oversegmentation near the edge (until total dilation of the edge), contour displacement, modification of the image size... These experiments indicate that some measures are not appropriate for the evaluation of edge detection for each type of boundary, and an ideal measure must be one that reacts as coherently possible to reality. Therefore, this paper shows the relevance of a boundary detection assessment that takes into consideration the distance of both the false positive and the false negative points created or missed by the boundary detection process.

The remainder of this paper is organized as follows. Section III is devoted to an overview of error measures based on statistics. Next, Section III reviews most existing referencebased edge measures involving distances of erroneous points, then points out the advantages and drawbacks of different measures, followed by the presentation of a new measure. Section IV presents experimental results for different synthetic data, and then quality measure results for real images. Finally, Section $\mathrm{V}$ gives perspectives for future work and draws the conclusions of the study.

\section{CONFUSION MATRIX-BASED ERROR ASSESSMENTS}

To assess an algorithm, the confusion matrix remains a cornerstone in boundary detection evaluation methods. Let $G_{t}$ be the reference contour map corresponding to ground truth and $D_{c}$ the detected contour map of an image $I$. Comparing pixel per pixel $G_{t}$ and $D_{c}$, the first criterion to be assessed is the common presence of edge/non-edge points. A basic evaluation is compounded from statistics resulting from a confusion matrix. To that effect, $G_{t}$ and $D_{c}$ are combined. Afterwards, denoting $|\cdot|$ as the cardinality of a set, all points are divided into four sets:

- True Positive points (TPs), common points of $G_{t}$ and $D_{c}$ : $T P=\left|D_{c} \cap G_{t}\right|$,

- False Positive points (FPs), spurious detected edges, i.e. erroneous pixels of $D_{c}$ and not in $G_{t}$ defined as boundary: $F P=\left|D_{c} \cap \neg G_{t}\right|$,

- False Negative points (FNs), missing boundary points of $D_{c}$, i.e. holes in the true contour: $F N=\left|\neg D_{c} \cap G_{t}\right|$,

- True Negative points (TNs), common non-edge points of $G_{t}$ and $D_{c}: T N=\left|\neg D_{c} \cap \neg G_{t}\right|$.

On the one hand, let us consider boundary detection of natural images, FPs appear in the presence of noise, texture or other contours influencing the filter used by the edge detection operator. On the other hand, FNs represent holes in a contour of $D_{c}$ (generally caused by blurred edges in the original image $I)$. For example, an incorrect threshold of the segmentation could generate both FPs and FNs. In the experiment, TPs, FPs and FNs are respectively represented in green, red and blue: see illustrations in Fig. 2 for more details. Computing only FPs and FNs enables a segmentation assessment to be performed [35][36]. Yet, combining at least these two quantities enables

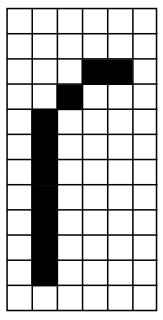

(a) $G_{t}$

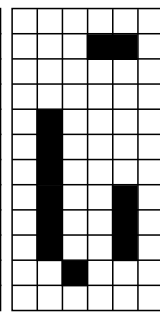

(b) $D_{c}$

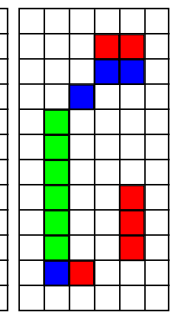

(c) $G_{t} \cup D_{c}$

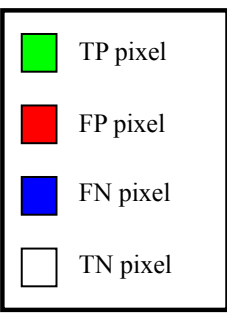

(d) Legend
Fig. 2. Illustration of TP (green), FP (red) and FN (blue) points. In (b), $D_{c}$ is contaminated with 6 FPs ans 4 FNs, illustrated with colors in (c). 
TABLE I

LIST OF ERROR MEASURES INVOLVING ONLY STATISTICS.

\begin{tabular}{lcc}
\hline Error measure name & Formulation & Parameters \\
\hline Binary noise-to-signal ratio [58] & $B_{S N R}\left(G_{t}, D_{c}\right)=\sqrt{\frac{\left|D_{c}\right|}{F P+F N}}$ & None \\
\hline $\begin{array}{l}\text { Complemented Performance } \\
\text { [51] [19] }\end{array}$ & $P_{m}^{*}\left(G_{t}, D_{c}\right)=1-\frac{T P}{T P+F P+F N}$ & None \\
\hline Segmentation Success Ratio [55] & $S S R\left(G_{t}, D_{c}\right)=1-\frac{T P^{2}}{\left|G_{t}\right| \cdot\left|D_{c}\right|}$ & None \\
\hline Complemented $\Phi$ measure [56] & $\Phi^{*}\left(G_{t}, D_{c}\right)=1-\frac{T P R \cdot T N}{T N+F P}$ & None \\
\hline Complemented $\chi^{2}$ measure [60] & $\chi^{2 *}\left(G_{t}, D_{c}\right)=1-\frac{T P R-T P-F P}{1-T P-F P} \cdot \frac{T P+F P+F P R}{T P+F P}$ & None \\
\hline Complemented $F_{\alpha}$ measure [39] & $F_{\alpha}^{*}\left(G_{t}, D_{c}\right)=1-\frac{P R E C \cdot T P R}{\alpha \cdot T P R+(1-\alpha) \cdot P R E C}, \quad$ with PREC $=\frac{T P}{T P+F P}$ & $\alpha \in] 0 ; 1]$ \\
\hline
\end{tabular}

a segmented image to be assessed more precisely. Thus, we present a list of statistical measures -others are detailed in [3], and a good-quaity edge detection method should obtain the smallest response for the three following indicators [2] [9]:

$$
\left\{\begin{aligned}
\text { Over-detection error : } \operatorname{Over}\left(G_{t}, D_{c}\right) & =\frac{F P}{|I|-\left|G_{t}\right|}, \\
\text { Under-detection error : } \operatorname{Under}\left(G_{t}, D_{c}\right) & =\frac{F N}{\left|G_{t}\right|}, \\
\text { Localization-error [29]: } \operatorname{Loc}\left(G_{t}, D_{c}\right) & =\frac{F P+F N}{|I|} .
\end{aligned}\right.
$$

One of the pioneer works in quantitative edge evaluation, reported by Deutsch and Fram [12] computed two parameters $P_{1}$ and $P_{2}$. The first evaluates the distribution of false positive points in function of the number of columns containing edge points, whereas $P_{2}$ quantifies the edge detection by counting the missing points for each line of $D_{c}$. Thus, $P_{1}$ and $P_{2}$ parameters are given by:

$$
P_{1}\left(G_{t}, D_{c}\right)=1-\frac{n_{\text {sig }}}{n_{\text {sig }}+\left(n_{\text {noise }}+F P\right) \cdot \frac{w_{\text {stan }} \cdot\left|G_{t}\right|}{w_{1} \cdot|I|}}
$$

and

$$
P_{2}\left(G_{t}, D_{c}\right)=1-\frac{\frac{n_{r}}{w_{2}}-\left(1-\left[1-\frac{n_{\text {noise }}}{\left|G_{t}\right|}\right]^{w_{1}}\right)}{\left[1-\frac{n_{\text {noise }}}{\left|G_{t}\right|}\right]^{w_{1}}},
$$

with:

$$
\left\{\begin{aligned}
n_{\text {noise }} & =\frac{F P \cdot\left|G_{t}\right|}{T N+F P} \\
n_{\text {sig }} & =\frac{T P-n_{\text {noise }}}{1-n_{\text {noise }}},
\end{aligned}\right.
$$

where $n_{r}$ and $w_{1}$ represent respectively the number of rows and columns in $D_{c}$ which contains a least one edge point (FP or TP point). Also, $w_{\text {stan }}$ corresponds to the number of columns of $G_{t}$ (which is the same as $D_{c}$ ), $w_{2}$ is the number of rows of the envelop rectangle containing $D_{c}$. These two parameters are close to 0 when the segmentation is efficient and tend towards one for poor detection. However, $P_{1}$ and $P_{2}$ would be more suited for the evaluation of vertical edges (see Fig. 3). On the contrary, the score of $P_{1}$ can be over 1 when $w_{1} \ll w_{\text {stan }}$, as in Fig. 5 Moreover, concerning $P_{2}$, when $n_{r}=w_{2}$, so $\frac{n_{r}}{w_{2}}=1$ and $P_{2}\left(G_{t}, D_{c}\right)=1-\frac{\left(1-n_{\text {noise }} /\left|G_{t}\right|\right)^{w_{1}}}{\left(1-n_{\text {noise }} /\left|G_{t}\right|\right)^{w_{1}}}=0$, translating, wrongly, a perfect segmentation, as in Fig. 3 (b), (c), (e), (f) and Fig. 5
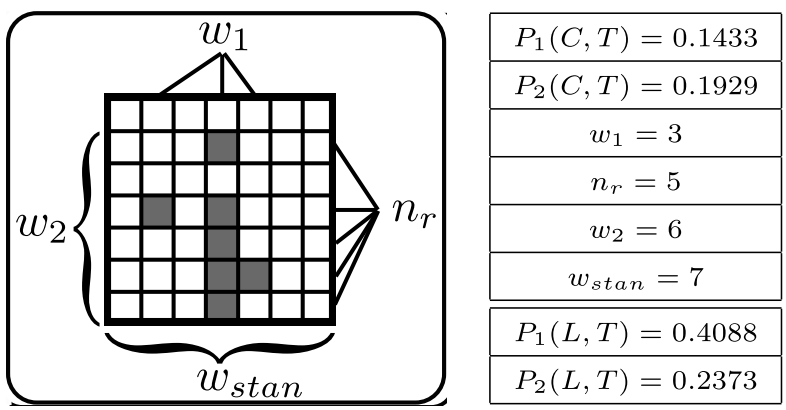

(a) $T$

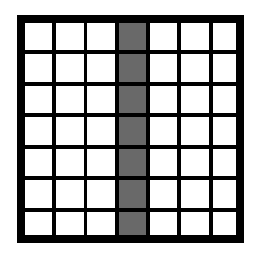

(b) $C$

$P_{1}(L, C)=1.0244$ $P_{2}(L, C)=0$

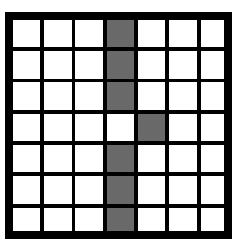

(e) $C_{2}$

$P_{1}\left(C, C_{2}\right)=0.091$

$P_{2}\left(C, C_{2}\right)=0$

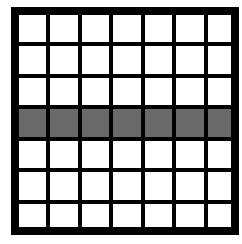

(c) $L$ $P_{1}(C, L)=1.200$ $P_{2}(C, L)=0$

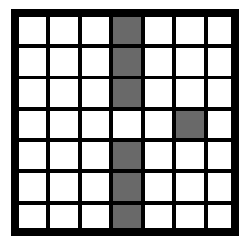

(f) $C_{3}$

$P_{1}\left(C, C_{3}\right)=0.091$

$P_{2}\left(C, C_{3}\right)=0$

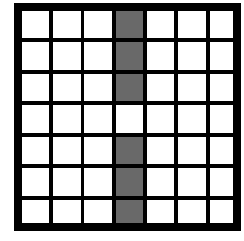

(d) $C_{1}$ $P_{2}\left(C, C_{1}\right)=0.1429$

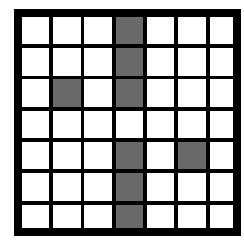

(g) $C_{4}$

$P_{1}\left(C, C_{4}\right)=0.121$

$P_{2}\left(C, C_{4}\right)=0.1654$ $P_{1}\left(C, C_{1}\right)=0$

Fig. 3. Computation of parameters $P_{1}$ and $P_{2}$ using contour images $7 \times 7$. The $C$ image in (b) is considerate as the $G_{t}$ image. $P_{1}$ penalizes $D_{c}$ having FPs whereas $P_{2}$ penalizes vertical contours with hole(s). In (d), $P_{1}\left(C, C_{1}\right)=0$ because $P_{1}$ records only FPs. Note in (a) that $P_{1}(L, C) \neq P_{1}(C, L)$. 
Several edge detection evaluations involving confusion matrices are presented in Table 1 Only TPs are an indicator, as for $S S R$, this measure is normalized in function of $\left|G_{t}\right| \cdot\left|D_{c}\right|$ and decreases with improved quality of detection, with $S S R=0$ qualifying a perfect segmentation.

The Binary Signal-to-Noise Ratio [58] $B_{S N R}$ (see table I] is inspired by the Signal-to-Noise Ratio $(S N R)$. It corresponds to a measure comparing the level of a signal in a reference signal to the level of the noise in a desired signal. Thus, $B_{S N R}$ computes a global error in function of the FPs and FNs; the fewer the numbers of FPs and FNs, the more the score increases and tends to the infinity when $G_{t}=D_{c}$.

Finally, the complemented Performance measure $P_{m}^{*}$ presented in table 1$]$ considers directly and simultaneously the three entities $T P, F P$ and $F N$ to assess a binary image [19]. The measure is normalized and decreases with improved quality of detection, with $P_{m}^{*}=0$ qualifying perfect segmentation.

By combining $F P, F N, T P$ and $T N$, another way to display evaluations is to create Receiver Operating Characteristic (ROC) [7] curves or Precision-Recall (PR) [39], involving True Positive Rates (TPR) and False Positive Rates (FPR):

$$
\left\{\begin{aligned}
\mathrm{TPR} & =\frac{T P}{T P+F N} \\
\mathrm{FPR} & =\frac{F P}{F P+T N} .
\end{aligned}\right.
$$

Derived from $T P R$ and $F P R$, the three measures $\Phi, \chi^{2}$ and $F_{\alpha}$ (detailed in Table $\mathbb{I}$ ) are frequently used in edge detection assessment. Using the complement of these measures results in a score close to 1 indicating good segmentation, and a score close to 0 indicating poor segmentation. Among these three measures, $F_{\alpha}$ remains the most stable because it does not consider the TNs, which are dominant in edge maps. Indeed, taking into consideration $T N$ in $\Phi$ and $\chi^{2}$ influences solely the measurement (as is the case in huge images).

These measures evaluate the comparison of two edge images, pixel per pixel, tending to severely penalize an (even slightly) misplaced contour. Furthermore, they depend heavily of the reference image $G_{t}$ which could be interpreted in different ways, as the different contours presented in Fig. 1 So statistical measures do not indicate enough significant variations of the desired contour shapes in the course of an evaluation (as illustrated in Fig. 5). As this penalization tends to be too severe, some evaluations resulting from the confusion matrix recommend incorporating spatial tolerance, particularly for the assimilation of TPs [48] [7] [39]. This inclusion could be carried by a distance threshold or a dilation of $D_{c}$ and/or $G_{t}$, as in [10] (see Eq. (58)). Such a strategy of assimilation leads to counting several near contours as stripes parallel to the desired boundary (issued from the edge detector itself or a blur/texture in the original image). For example, awarding a spatial tolerance for the FPs will ensure the same score for an over-segmentation near the edges (experiments illustrated in Fig. 13), if the spatial tolerance stays greater than the FP distances from the true pixel. Tolerating a distance from the true contour and integrating several TPs for one detected contour are opposite to the principle of unicity in edge detection expressed by the 3rd Canny criterion: an optimal edge detector must produce a single response for one contour [8]. Thus, from the discussion below, only one FP or one TP should be considered in the boundary detection evaluation process. Finally, to perform an edge evaluation, the assessment should penalize a misplaced edge point proportionally to the distance from its true location.

\section{ASSESSMENT INVOLVING DISTANCES OF MISPLACED PIXELS}

A reference-based edge map quality measure requires that a displaced edge should be penalized in function not only of FPs and/or FNs but also of the distance from the position where it should be located. Table $\Pi$ reviews the most relevant measures in the literature with a new one called $\Psi$. Some distance measures are specified in the evaluation of over-segmentation (i.e. presence of FPs) and others in the assessment of under segmentation (i.e. missing ground truth points). A complete edge detection evaluation measure takes into account both under- and over-segmentation assessment, the studied measures are detailed in the following subsection.

\section{A. Existing quality measures involving distances}

The common feature between these evaluators corresponds to the error distance $d_{G_{t}}(p)$ or/and $d_{D_{c}}(p)$. Indeed, for a pixel belonging to the desired contour $p \in D_{c}, d_{G_{t}}(p)$ represents the minimal distance between $p$ and $G_{t}$. On the contrary, if a pixel $p$ belongs to the ground truth $G_{t}, d_{D_{c}}(p)$ is the minimal distance between $p$ and $D_{c}$. Mathematically, denoting $\left(x_{p}, y_{p}\right)$ and $\left(x_{t}, y_{t}\right)$ the pixel coordinates of two points $p$ and $t$ respectively, thus $d_{G_{t}}(p)$ and $d_{D_{c}}(p)$ are described by:

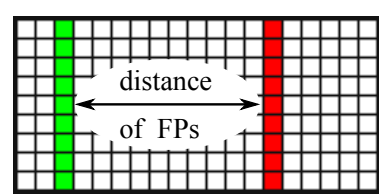

(a) Vertical edge lines with false positive points

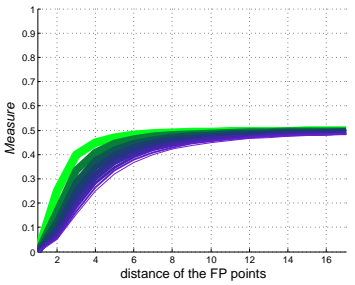

(b) FoM scores

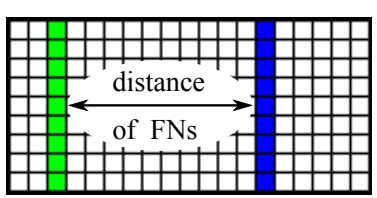

(c) Vertical edge line with false negative points

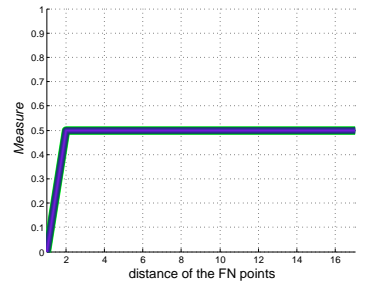

(d) FoM scores

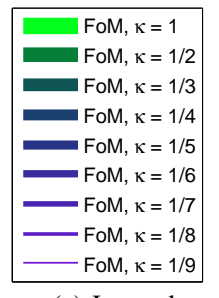

(e) Legend

Fig. 4. Evolution of FoM in function of the the distance of the false positive/negative points and $\kappa$ parameter. A vertical line of false positive points (a) or false negative points (c) is shifted by a maximum distance of 16 pixels and the measured scores are plotted in function of the displacement of this distance. 
TABLE II

LIST OF ERROR MEASURES COMPARED IN THIS WORK. IN THE LITERATURE, THE MOST COMMON VALUES ARE $k=1$ OR $k=2$.

\begin{tabular}{|c|c|c|}
\hline Error measure name & Formulation & Parameters \\
\hline $\begin{array}{l}\text { Figure of Merit }(F o M) \\
{[1]}\end{array}$ & $F o M\left(G_{t}, D_{c}\right)=1-\frac{1}{\max \left(\left|G_{t}\right|,\left|D_{c}\right|\right)} \cdot \sum_{p \in D_{c}} \frac{1}{1+\kappa \cdot d_{G_{t}}^{2}(p)}$ & $\kappa \in] 0 ; 1]$ \\
\hline $\begin{array}{l}\text { FoM of over- } \\
\text { segmentation [52] }\end{array}$ & $F_{o M_{e}}\left(G_{t}, D_{c}\right)=1-\frac{1}{\max \left(\mathrm{e}^{-F P}, F P\right)} \cdot \sum_{p \in D_{c} \cap \neg G_{t}} \frac{1}{1+\kappa \cdot d_{G_{t}}^{2}(p)}$ & $\kappa \in] 0 ; 1]$ \\
\hline FoM revisited 47] & $F\left(G_{t}, D_{c}\right)=1-\frac{1}{\left|G_{t}\right|+\beta \cdot F P} \cdot \sum_{p \in G_{t}} \frac{1}{1+\kappa \cdot d_{D_{c}}^{2}(p)}$ & $\begin{array}{l}\kappa \in] 0 ; 1] \text { and } \beta \in \\
\mathbb{R}^{+}\end{array}$ \\
\hline $\begin{array}{l}\text { Combination of FoM and } \\
\text { statistics [5] }\end{array}$ & $d_{4}\left(G_{t}, D_{c}\right)=\frac{1}{2} \cdot \sqrt{\frac{\left(T P-\max \left(\left|G_{t}\right|,\left|D_{c}\right|\right)\right)^{2}+F N^{2}+F P^{2}}{\left(\max \left(\left|G_{t}\right|,\left|D_{c}\right|\right)\right)^{2}}+F o M\left(G_{t}, D_{c}\right)}$ & $\begin{array}{l}\kappa \in] 0 ; 1] \text { and } \beta \in \\
\mathbb{R}^{+}\end{array}$ \\
\hline $\begin{array}{l}\text { Edge map quality measure } \\
\text { 433 }\end{array}$ & $D_{p}\left(G_{t}, D_{c}\right)=\frac{1 / 2}{|I|-\left|G_{t}\right|} \cdot \sum_{p \in D_{c}}\left(1-\frac{1}{1+\kappa \cdot d_{G_{t}}^{2}(p)}\right)+\frac{1 / 2}{\left|G_{t}\right|} \cdot \sum_{p \in G_{t}}\left(1-\frac{1}{1+\kappa \cdot d_{G_{t} \cap D_{c}}^{2}(p)}\right)$ & $\kappa \in] 0 ; 1]$ \\
\hline Yasnoff measure [59] & $\Upsilon\left(G_{t}, D_{c}\right)=\frac{100}{|I|} \cdot \sqrt{\sum_{p \in D_{c}} d_{G_{t}}^{2}(p)}$ & None \\
\hline Hausdorff distance [24] & $H\left(G_{t}, D_{c}\right)=\max \left(\max _{p \in D_{c}} d_{G_{t}}(p), \max _{p \in G_{t}} d_{D_{c}}(p)\right)$ & None \\
\hline $\begin{array}{l}\text { Distance to } G_{t} \\
{[24][3][31]}\end{array}$ & $D^{k}\left(G_{t}, D_{c}\right)=\frac{1}{\left|D_{c}\right|} \cdot \sqrt[k]{\sum_{p \in D_{c}} d_{G_{t}}^{k}(p)}, \quad k=1$ for 46] & $k \in \mathbb{R}^{+}$ \\
\hline Maximum distance 13 & $f_{2} d_{6}\left(G_{t}, D_{c}\right)=\max \left(\frac{1}{\left|D_{c}\right|} \cdot \sum_{p \in D_{c}} d_{G_{t}}(p), \frac{1}{\left|G_{t}\right|} \cdot \sum_{p \in G_{t}} d_{D_{c}}(p)\right)$ & None \\
\hline $\begin{array}{l}\text { Oversegmentation } \\
{[20] 40}\end{array}$ & $\Theta\left(G_{t}, D_{c}\right)=\frac{1}{F P} \cdot \sum_{p \in D_{c}}\left(\frac{d_{G_{t}}(p)}{\delta_{T H}}\right)^{k}, \quad k=\delta_{T H}=1$ for [20] & $\begin{array}{l}\text { for }[40]: k \in \mathbb{R}^{+} \text {and } \\
\delta_{T H} \in \mathbb{R}_{*}+\end{array}$ \\
\hline $\begin{array}{l}\text { Undersegmentation } \\
{[20[40]}\end{array}$ & $\Omega\left(G_{t}, D_{c}\right)=\frac{1}{F N} \cdot \sum_{p \in G_{t}}\left(\frac{d_{D_{c}}(p)}{\delta_{T H}}\right)^{k}, \quad k=\delta_{T H}=1$ for [20] & $\begin{array}{l}\text { for [40]: } k \in \mathbb{R}^{+} \text {and } \\
\delta_{T H} \in \mathbb{R}_{*}^{+}\end{array}$ \\
\hline $\begin{array}{l}\text { Baddeley's Delta Metric } \\
{[2]}\end{array}$ & $\Delta^{k}\left(G_{t}, D_{c}\right)=\sqrt[k]{\frac{1}{|I|} \cdot \sum_{p \in I}\left|w\left(d_{G_{t}}(p)\right)-w\left(d_{D_{c}}(p)\right)\right|^{k}}$ & $\begin{array}{l}k \in \mathbb{R}^{+} \text {and a con- } \\
\text { vex function } w: \\
\mathbb{R} \mapsto \mathbb{R}\end{array}$ \\
\hline $\begin{array}{l}\text { Symmetric distance } \\
\text { [13] 31 }\end{array}$ & $S^{k}\left(G_{t}, D_{c}\right)=\sqrt[k]{\frac{\left.\sum_{p \in D_{c}} d_{G_{t}}^{k}(p)\right)+\sum_{p \in G_{t}} d_{D_{c}}^{k}(p)}{\left|D_{c} \cup G_{t}\right|}}$, & $k \in \mathbb{R}^{+}$ \\
\hline $\begin{array}{l}\text { Magnier et al. measure } \\
\text { [37] }\end{array}$ & $\Gamma\left(G_{t}, D_{c}\right)=\frac{F P+F N}{\left|G_{t}\right|^{2}} \cdot \sqrt{\sum_{p \in D_{c}} d_{G_{t}}^{2}(p)}$ & None \\
\hline $\begin{array}{l}\text { Symmetric distance mea- } \\
\text { sure }\end{array}$ & $\Psi\left(G_{t}, D_{c}\right)=\frac{F P+F N}{\left|G_{t}\right|^{2}} \cdot \sqrt{\sum_{p \in G_{t}} d_{D_{c}}^{2}(p)+\sum_{p \in D_{c}} d_{G_{t}}^{2}(p)}$ & None \\
\hline
\end{tabular}

$$
\left\{\begin{array}{l}
\text { for } p \in D_{c}: \\
d_{G_{t}}(p)=\operatorname{Inf}\left\{\sqrt{\left(x_{p}-x_{t}\right)^{2}+\left(y_{p}-y_{t}\right)^{2}}, t \in G_{t}\right\}, \\
\text { for } p \in G_{t}: \\
d_{D_{c}}(p)=\operatorname{Inf}\left\{\sqrt{\left(x_{p}-x_{t}\right)^{2}+\left(y_{p}-y_{t}\right)^{2}}, t \in D_{c}\right\} .
\end{array}\right.
$$

These distances refer to the Euclidean distance, even though some authors include other types of distance, see [31]. The measures presented in Table $\Pi$ are tuned in function of the various parameters referenced in Table IV] After marking a theoretical comparison of all these edge detection assessments and carrying out in depth comparison of their parameters, the experimental results are presented.

1) Figure of Merit and its derivatives: First, to achieve a quantitative index of edge detector performance, one of the most popular descriptors is the Figure of Merit $(F o M)$. This distance measure ranges from 0 to 1 , where 0 corresponds to a perfect segmentation [1]. The constant value $\kappa$ is fixed at $1 / 9$, $1 / 4$ or $1 / 10$ (the last value is the one used in the experiments of this paper). The more $\kappa$ is close to 1 , the more $F_{O M}$ tackles FPs, as illustrated in Fig 4 Widely utilized for comparing several different segmentation methods, in particular thanks to its normalization criterion, this assessment approach nonetheless suffers from a main drawback. Whenever FNs are created, for example a contour chain (even long) which is not totally extracted, the distance of FNs $\left(d_{D_{c}}(p)\right)$ are not recorded. Indeed, FoM can be rewritten as:

$F o M\left(G_{t}, D_{c}\right)=1-\frac{\sum_{p \in D_{c} \cap G_{t}} \frac{1}{1+\kappa \cdot d_{G_{t}}^{2}(p)}+\sum_{p \in D_{c} \cap \neg G_{t}} \frac{1}{1+\kappa \cdot d_{G_{t}}^{2}(p)}}{\max \left(\left|G_{t}\right|,\left|D_{c}\right|\right)}$, 


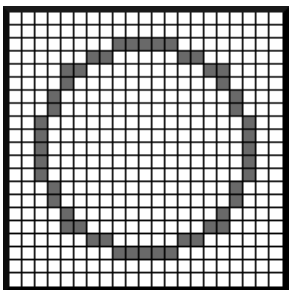

(a) $G_{t},\left|G_{t}\right|=48$

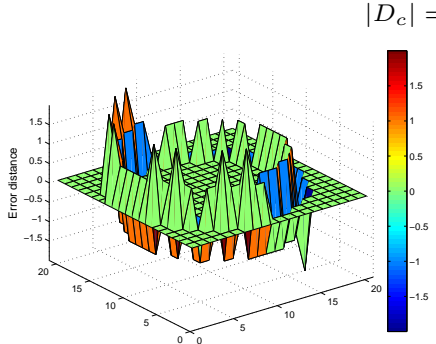

(d) Error distance: $G_{t}$ v.s. $D_{1}$

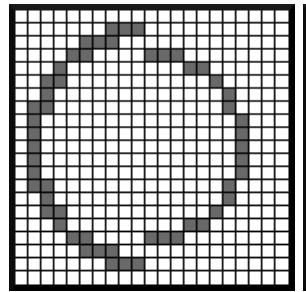

(b) $D_{1}, F P=54$

$\left|D_{c}\right|=54$

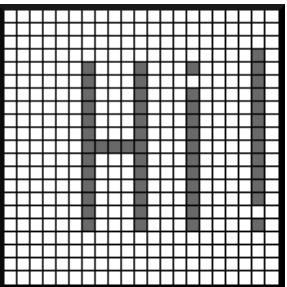

$\left|D_{c}\right|=54$ (c) $D_{2}, F P=54$

\begin{tabular}{|c|c|}
\hline Over $\left(G_{t}, D_{\{1,2\}}\right)=0.12$ & Under $\left(G_{t}, D_{\{1,2\}}\right)=1$ \\
\hline $\operatorname{Loc}\left(G_{t}, D_{\{1,2\}}\right)=0.24$ & $B_{S N R}\left(G_{t}, D_{\{1,2\}}\right)=0.73$ \\
\hline$P_{m}^{*}\left(G_{t}, D_{\{1,2\}}\right)=1$ & $\Phi^{*}\left(G_{t}, D_{\{1,2\}}\right)=1$ \\
\hline$\chi^{2 *}\left(G_{t}, D_{\{1,2\}}\right)=0.98$ & $F_{\alpha=0.5}^{*}\left(G_{t}, D_{\{1,2\}}\right)=1$ \\
\hline \hline$P_{1}\left(G_{t}, D_{1}\right)=16.25$ & $P_{1}\left(G_{t}, D_{2}\right)=1.63$ \\
\hline$P_{2}\left(G_{t}, D_{1}\right)=0$ & $P_{2}\left(G_{t}, D_{2}\right)=0$ \\
\hline \hline$F o M\left(G_{t}, D_{1}\right)=0.22$ & $F o M\left(G_{t}, D_{2}\right)=0.60$ \\
\hline$F o M_{e}\left(G_{t}, D_{1}\right)=0.22$ & $F o M_{e}\left(G_{t}, D_{2}\right)=0.60$ \\
\hline$F\left(G_{t}, D_{1}\right)=0.63$ & $F\left(G_{t}, D_{2}\right)=0.75$ \\
\hline$d_{4}\left(G_{t}, D_{1}\right)=0.84$ & $d_{4}\left(G_{t}, D_{2}\right)=0.89$ \\
\hline$D_{p}\left(G_{t}, D_{1}\right)=0.51$ & $D_{p}\left(G_{t}, D_{2}\right)=0.54$ \\
\hline$S F_{o} M\left(G_{t}, D_{1}\right)=0.25$ & $S F_{o M}\left(G_{t}, D_{2}\right)=0.40$ \\
\hline$M F O M\left(G_{t}, D_{1}\right)=0.29$ & $M F o M\left(G_{t}, D_{2}\right)=0.40$ \\
\hline$\Upsilon\left(G_{t}, D_{1}\right)=12.99$ & $\Upsilon\left(G_{t}, D_{2}\right)=37.34$ \\
\hline$H\left(G_{t}, D_{1}\right)=1.41$ & $H\left(G_{t}, D_{2}\right)=7.67$ \\
\hline$H_{5 \%}\left(G_{t}, D_{1}\right)=1.41$ & $H_{5 \%}\left(G_{t}, D_{2}\right)=6.71$ \\
\hline$D_{k=1}^{k}\left(G_{t}, D_{1}\right)=1.06$ & $D_{k=1}^{k}\left(\left(G_{t}, D_{2}\right)=3.05\right.$ \\
\hline$D_{k=2}^{k}\left(G_{t}, D_{1}\right)=0.16$ & $D_{k=2}^{k}\left(\left(G_{t}, D_{2}\right)=0.48\right.$ \\
\hline$f_{2} d_{6}\left(G_{t}, D_{1}\right)=1.06$ & $f_{2} d_{6}\left(G_{t}, D_{2}\right)=3.05$ \\
\hline$\Theta_{\delta_{T H}=1}\left(G_{t}, D_{1}\right)=1.19$ & $\Theta_{\delta_{T H}=1}\left(G_{t}, D_{2}\right)=12.26$ \\
\hline$\Theta_{\delta_{T H}=5}\left(G_{t}, D_{1}\right)=0.05$ & $\Theta_{\delta_{T H}=5}\left(G_{t}, D_{2}\right)=0.49$ \\
\hline$\Omega_{\delta_{T H}=1}\left(G_{t}, D_{1}\right)=1.04$ & $\Omega_{\delta_{T H}=1}\left(G_{t}, D_{2}\right)=5.12$ \\
\hline$\Omega_{\delta_{T H}=5}\left(G_{t}, D_{1}\right)=0.04$ & $\Omega_{\delta_{T H}=5}\left(G_{t}, D_{2}\right)=0.20$ \\
\hline$\Delta_{w}^{k}\left(G_{t}, D_{1}\right)=0.96$ & $\Delta_{w}^{k}\left(G_{t}, D_{2}\right)=2.31$ \\
\hline$S D_{k=1}^{k}\left(G_{t}, D_{1}\right)=1.04$ & $S D_{k=1}^{k}\left(\left(G_{t}, D_{2}\right)=2.57\right.$ \\
\hline$S D_{k=1}^{k}\left(G_{t}, D_{1}\right)=1.05$ & $S D_{k=2}^{k}\left(\left(G_{t}, D_{2}\right)=2.98\right.$ \\
\hline$\Gamma\left(G_{t}, D_{1}\right)=0.34$ & $\Gamma\left(G_{t}, D_{2}\right)=0.57$ \\
\hline$\Psi\left(G_{t}, D_{1}\right)=0.46$ & $\Psi\left(G_{t}, D_{2}\right)=0.72$ \\
\hline \hline$K P I_{\Gamma}\left(G_{t}, D_{1}\right)=0.16$ & $K P I_{\Gamma}\left(G_{t}, D_{2}\right)=0.27$ \\
\hline$K P I_{\Psi}\left(G_{t}, D_{1}\right)=0.22$ & $K P I_{\Psi}\left(G_{t}, D_{2}\right)=0.37$ \\
\hline & \\
\hline
\end{tabular}

Fig. 5. Results of evaluation measures. For the two candidate images, the number of FPs and number of FNs are the same: FPs: $\left|D_{1} \cap \neg G_{t}\right|=\left|D_{2} \cap \neg G_{t}\right|=54$ and FNs: $\left|\neg D_{1} \cap G_{t}\right|=\left|\neg D_{2} \cap G_{t}\right|=$ $\left|G_{t}\right|=48$. Also, $D_{1} \cap G_{t}=D_{2} \cap G_{t}=\varnothing$, so $T P=0$ and $S S R\left(G_{t}, D_{1}\right)=$ $\operatorname{SSR}\left(G_{t}, D_{2}\right)=1$. The assessments involving distances of FPs and/or FNs heavily penalize $D_{c}$, including misplaced points with greater distances. hence:

$$
F o M\left(G_{t}, D_{c}\right)=1-\frac{T P+\sum_{p \in D_{c} \cap \neg G_{t}} \frac{1}{1+\kappa \cdot d_{G_{t}}^{2}(p)}}{\max \left(\left|G_{t}\right|,\left|D_{c}\right|\right)},
$$

because, for $p \in D_{c} \cap G_{t}, d_{G_{t}}^{2}(p)=0$ and $\frac{1}{1+\kappa \cdot d_{G_{t}}^{2}(p)}=1$. Knowing that $T P=\left|G_{t}\right|-F N$, for the extreme cases, the FoM measures takes the following values:

$$
\left\{\begin{array}{l}
\text { if } F P=0: \\
F o M\left(G_{t}, D_{c}\right)=1-\frac{T P}{\left|G_{t}\right|}, \\
\text { if } F N=0: \\
F o M\left(G_{t}, D_{c}\right)=1-\frac{1}{\max \left(\left|G_{t}\right|,\left|D_{c}\right|\right)} \cdot \sum_{p \in D_{c} \cap \neg G_{t}} \frac{1}{1+\kappa \cdot d_{G_{t}}^{2}(p)} .
\end{array}\right.
$$

Consequently, FoM counts only TPs to penalize FN points (Fig 4 (d)) whereas only distances of FPs are recorded (Fig 4 (b)). Moreover, for $F P>0$, as $\frac{1}{1+\kappa \cdot d_{G_{t}}^{2}(p)}<1$, it can be easily demonstrate that the FoM measure penalizes the over-detection very low compared to the under-detection. The curve in Fig. 6 shows that when $F N>F P$, the penalization of missing points (FNs) becomes higher wheareas it is weak $F N<F P$. Incidentally, $F_{o} M_{e}$ represents an extension of FoM by counting only FPs [52], strictly evaluating the oversegmentation. In fact, it computes a mean of $\frac{1}{1+\kappa \cdot d_{G_{t}}^{2}(p)}$ for all the FPs. Consequently, in the presence or absence of FNs, a contour image is considered by the $F o M_{e}$ criterion as correctly segmented. The experiments in Fig. 6, Fig. 8 and Section IV illustrate this undesirable behavior. Some improvements have been developed, such as $F$ and $d_{4}$. In order to overcome the gaps in FoM, F computes the distances of FNs from $G_{t}$ and the measure is tuned by the number of FPs and the cardinality of $G_{t}$ (choosing $\beta=1$ [47]). As

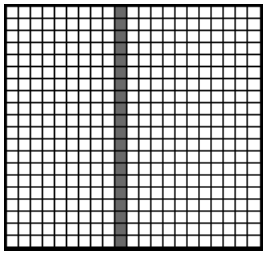

(a) Image $G_{t}$,

$\left|G_{t}\right|=21$

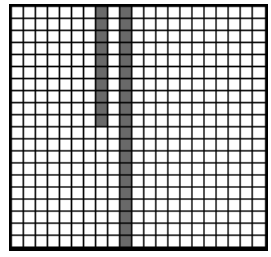

(b) Image $L_{1}$, $\left|D_{c}\right|=31, T P=, 21$ $F P=10, F N=0$

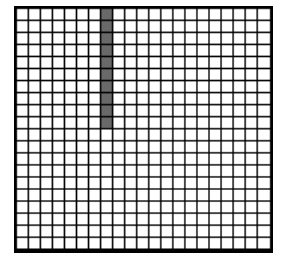

(c) Image $L_{2}$

$\left|D_{c}\right|=10, T P=0$, $F P=10, F N=21$

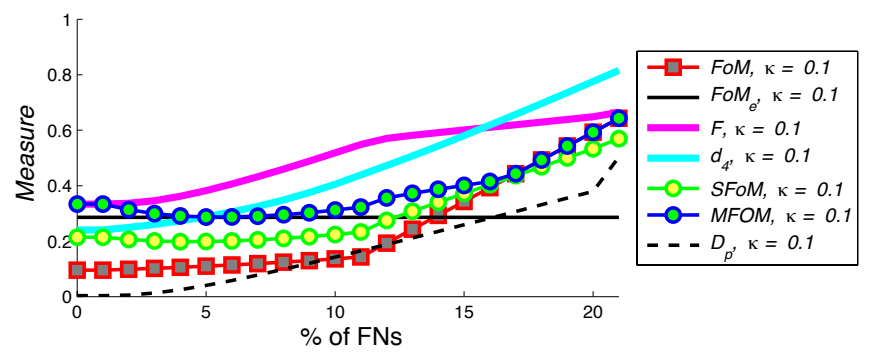

(d) Scores of FoM and its derived measures.

Fig. 6. Results of FoM and its derived measures in function of the $\%$ of FNs, comparing $G_{t}$ and $L_{1}$. When the number of FNs attains $100 \%$, the candidate edge image corresponds to $L_{2}$. 
TABLE III

BEHAVIOR OF $d_{4}$ MEASURE IN FUNCTION OF DIFFERENT SEGMENTATION LEVELS, NOTING $M=\left(\max \left(\left|G_{t}\right|,\left|D_{c}\right|\right)\right)^{2}$.

\begin{tabular}{|c|c|c|c|c|}
\hline Segmentation level & $F o M$ & $\frac{\left|T P-\max \left(\left|G_{t}\right|,\left|D_{c}\right|\right)\right|}{M}$ & $\frac{F N}{M}$ & $\frac{F P}{M}$ \\
\hline \hline Good & $\approx 0$ & $\approx 0$ & $\approx 0$ & $\approx 0$ \\
\hline$F P \nearrow, F N=0$ & $>0$ & $>0$ & 0 & $>0$ \\
\hline$F P=0, F N \nearrow$ & $>0$ & $>0$ & $>0$ & 0 \\
\hline$F P \nearrow, F N \nearrow$ & $>0$ & $>0$ & $>0$ & $>0$ \\
\hline
\end{tabular}

pointed out through Eq. 3 for $F o M$ and illustrated in Fig. 6. $F$ behaves inversely to $F o M$ concerning FPs/FNs points: $F$ is more sensitive to FPs than FNs. Also, $d_{4}$ represents another enhancement, this edge measure depends particularly on TP, FP, FN and FoM; $d_{4}$ is normalized with the $\frac{1}{2}$ coefficient [5]. Nonetheless, even though $d_{4}$ behaves correctly for the experiment in Fig. 6 this measure focuses on FPs and penalizes FNs like the $F o M$ measure, as detailed in Table III This measure suffers from two main drawbacks. On the one hand, the sum of all its terms yields a high sensibility to edge displacements. On the other hand, in the case of a pure undersegmentation, when $F N \rightarrow 0, d_{4}$ belongs to [0.7, 0.85] but does not attains the score of 1 .

As described here, an effective measure for the evaluation of the edge detection is not only directed by $d_{G_{t}}$ or $d_{D_{c}}$. Thus, inspired by $f_{2} d_{6}$ (see bottom and [13]), another way to avoid the computation of only the distance of FPs in FoM (or only the distance of FNs in $F$ ) is to consider the combination of both $\operatorname{FoM}\left(G_{t}, D_{c}\right)$ and $\operatorname{FoM}\left(D_{c}, G_{t}\right)$, as in the following two formulas:

- Symmetric Figure of Merit:

$\operatorname{SFoM}\left(G_{t}, D_{c}\right)=\frac{1}{2} \cdot \operatorname{FoM}\left(G_{t}, D_{c}\right)+\frac{1}{2} \cdot \operatorname{FoM}\left(D_{c}, G_{t}\right)$

- Maximum Figure of Merit:

$M F o M\left(G_{t}, D_{c}\right)=\max \left(F o M\left(G_{t}, D_{c}\right), \operatorname{FoM}\left(D_{c}, G_{t}\right)\right)$.

As $F o M$ is a normalized measure, $S F o M$ and $M F o M$ are also normalized. Finally, $S F o M$ and $M F o M$ take into account both distances of FNS (i.e. $d_{D_{c}}$ ) and FPs (i.e. $d_{G_{t}}$ ), so they can compute a global evaluation of a contour image. Nevertheless, Fig. 6 shows that $S F o M$ behaves like FoM when $F N>F P$ and that $M F O M$ is not monotonous for this experiment (whereas only FNs are added).

2) Hausdorff distance its enhancements: A second measure widely computed in matching techniques is represented by the Hausdorff distance $H$. In object recognition [22], the algorithm aims to minimize $H$, which measure the mismatch of two sets of points [24][45]. This max-min distance could be strongly deviated by only one pixel which can be positioned sufficiently far from the pattern (illustrated in Fig. 87; so the measured distance becomes that between the pattern and the (erroneous) point, in that case disturbing the score of $H$. To improve the measure such that $H$ becomes less sensitive to outliers, one idea is to compute $H$ with a proportion of the maximum distances (for example $5 \%, 10 \%$ or $15 \%$ of the values [24]); let us note $H_{n \%}$ this measure for $n \%$ of values

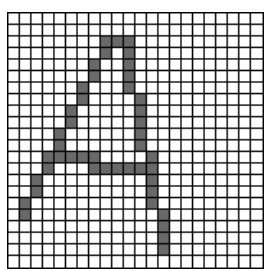

(a) Image $A$, $\left|G_{t}\right|=45$

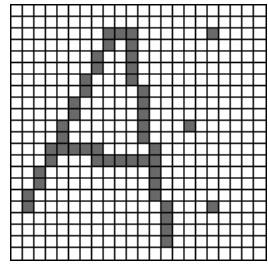

(b) Image $A_{n}$,

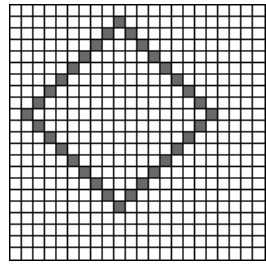

(c) Image $L$,

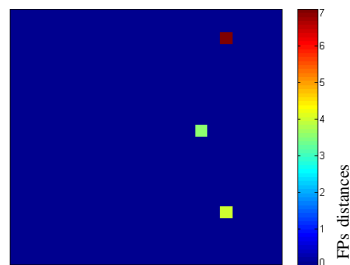

(d) Distance map: $A$ vs. $A_{n}$
$\left|D_{c}\right|=48, T P=, 45$ $F P=3, F N=0$
$\left|D_{c}\right|=32, T P=4$,

$F P=28, F N=41$

\begin{tabular}{|c|c|}
\hline$P_{1}\left(A, A_{n}\right)=0.01$ & $P_{1}(A, L)=0.80$ \\
\hline$P_{2}\left(A, A_{n}\right)=0$ & $P_{2}(A, L)=0$ \\
\hline$P_{m}^{*}\left(A, A_{n}\right)=0.06$ & $P_{m}^{*}(A, L)=0.95$ \\
\hline$S S R\left(A, A_{n}\right)=0.06$ & $S S R(A, L)=0.99$ \\
\hline$\Phi^{*}\left(A, A_{n}\right)=0.01$ & $\Phi^{*}(A, L)=0.92$ \\
\hline$\chi^{2 *}\left(A, A_{n}\right)=0.07$ & $\chi^{2 *}(A, L)=0.99$ \\
\hline$F_{\alpha=0.5}^{*}\left(A, A_{n}\right)=0.03$ & $F_{\alpha=0.5}^{*}(A, L)=0.90$ \\
\hline \hline$F o M\left(A, A_{n}\right)=0.05$ & $F o M(A, L)=0.60$ \\
\hline$F o M_{e}\left(A, A_{n}\right)=0.50$ & $F o M_{e}(A, L)=0.83$ \\
\hline$F\left(A, A_{n}\right)=0.06$ & $F(A, L)=0.66$ \\
\hline$d_{4}\left(A, A_{n}\right)=0.05$ & $d_{4}(A, L)=0.78$ \\
\hline$S F o M\left(A, A_{n}\right)=0.06$ & $S F o M(A, L)=0.53$ \\
\hline$M F o M\left(A, A_{n}\right)=0.06$ & $S F o M(A, L)=0.60$ \\
\hline$D_{p}\left(A, A_{n}\right)=0.003$ & $D_{p}(A, L)=0.29$ \\
\hline$\Upsilon\left(A, A_{n}\right)=2.93$ & $\Upsilon(A, L)=1.82$ \\
\hline$H\left(A, A_{n}\right)=7$ & $H(A, L)=5.83$ \\
\hline$H_{5 \%}\left(A, A_{n}\right)=5.5$ & $H_{5 \%}(A, L)=5.37$ \\
\hline$D^{k, k=1}\left(A, A_{n}\right)=0.30$ & $D^{k, k=1}((A, L)=2.05$ \\
\hline$D^{k, k=2}\left(A, A_{n}\right)=0.18$ & $D^{k, k=2}((A, L)=0.44$ \\
\hline$f_{2} d_{6}\left(A, A_{n}\right)=0.30$ & $f_{2} d_{6}(A, L)=2.11$ \\
\hline$\Theta_{\delta_{T H}=1}\left(A, A_{n}\right)=4.87$ & $\Theta_{\delta_{T H}=1}(A, L)=2.34$ \\
\hline$\Theta_{\delta_{T H}=3}\left(A, A_{n}\right)=2.89$ & $\Theta_{\delta_{T H}=3}(A, L)=0.79$ \\
\hline$\Omega_{\delta_{T H}=1}\left(A, A_{n}\right)=0$ & $\Omega_{\delta_{T H}=1}(A, L)=2.31$ \\
\hline$\Omega_{\delta_{T H}=3}\left(A, A_{n}\right)=0$ & $\Omega_{\delta_{T H}=3}(A, L)=0.77$ \\
\hline$\Delta^{k}\left(A, A_{n}\right)=2.11$ & $\Delta^{k}(A, L)=2.56$ \\
\hline$S D^{k, k=1}\left(A, A_{n}\right)=2.12$ & $S D^{k, k=1}(A, L)=2.09$ \\
\hline$S D^{k, k=2}\left(A, A_{n}\right)=2.71$ & $S D^{k, k=2}(A, L)=2.51$ \\
\hline$\Gamma\left(A, A_{n}\right)=0.01$ & $\Gamma(A, L)=0.48$ \\
\hline$\Psi\left(A, A_{n}\right)=0.01$ & $\Psi(A, L)=0.75$ \\
\hline \hline$K P I_{\Gamma}\left(A, A_{n}\right)=0.01$ & $K P_{\Gamma}(A, L)=0.24$ \\
\hline$K I_{\Psi}\left(A, A_{n}\right)=0.01$ & $K P I_{\Psi}(A, L)=0.39$ \\
\hline
\end{tabular}

Fig. 7. Results of evaluation measures. Even edge evaluations involving distances can be disturbed by few misplaced pixels and do not respect the shape of the true pattern.

$\left(n \in \mathbb{R}_{*}{ }^{+}\right)$. Even though a mean percentage of the distance does not guarantee an optimized comparison, as illustrated in Fig. 7, this enhancement helps with regard to robustness, and some other modifications are proposed in [61] and in [4].

Inspired by the Hausdorff distance with a view to developing 
a new method that is robust with regard to a small number of outliers, some researchers have proposed other measures and studied their behaviors in the presence of misplaced edge points [2] [13]. As $H_{n \%}$ remains close to the Hausdorff distance, the rank $n$ acts as a threshold for erroneous pixels and $H_{n \%}$ behaves as $H$. As pointed out in [13], an average distance from the edge pixels in the candidate image to those in the ground truth is more appropriate for matching purposes than $H$ and $H_{n \%}$. A first proposition of this distance is $D^{k}$ which represents an error distance only in function of $d_{G_{t}}$. Also, the Yasnoff measure, called $\Upsilon$, seems to $D^{k}$, with $k=2$, using a different coefficient of $\frac{100}{|I|}$ [59]. The distance measures $D^{k}$ and $\Upsilon$ estimate the divergence of FPs; in other words, they correspond to a measure of over-segmentation. On the contrary, the sole use of a distance $d_{D_{c}}$ instead of $d_{G_{t}}$ enables an estimation of the FN divergences, representing an undersegmentation (as in $\Omega$ ). Precisely, $\theta$ and $\Omega$ represent two overand under-segmentation assessment measures, where $\delta_{T H}$ is the maximum distance allowed to search for a contour point (see also distortion rates in [18]). These distance measures penalize misplaced points further than $\delta_{T H}$ from $G_{t}$ [40]. Choosing, $\delta_{T H}>1$ is equivalent to taking into account the relative position for the over- and under-segmentation and goes back to the problem of the spatial tolerance detailed in Section II). Finally, as concluded in [9], a complete and optimum edge detection evaluation measure should combine assessments of both over- and under-segmentation, as $f_{2} d_{6}$ and $S^{k}$. Thus, the score of the $f_{2} d_{6}$ corresponds to the maximum between the over- and the under-segmentation whereas the values obtained by $S^{k}$ reprensents their mean. Moreover, $S^{k}$ takes small values in the presence of low level of outliers whereas the score becomes large as the level of mistaken points increases [13][31] but is sensitive to remote misplaced points as represented in Fig. 8

Combining both $d_{D_{c}}$ and $d_{G_{t}}$, Baddeley's Delta Metric $\left(\Delta^{k}\right)$ [2] is a measure derived from the Hausdorff distance which is intended to estimate the dissimilarity between each element of two binary images. Finally, as this distance measure is based on the mean difference between the two compared images and is useful in region segmentation [26]. For this measure, $w$ represents a weighting concave function, in general $w(x)=x$ or $w(x)=\min \left(\sqrt{n^{2}+m^{2}}, x\right)$ for an image of size $m \times n$, with $m$ and $n \in \mathbb{N}^{*}$ [30] (in our experiments, we use $w(x)=x$ ). Compared to automatic threshold algorithms such as [41], the threshold at which the edge detector obtains the best edge map is more appropriate when it is computed by the minimum value of $\Delta^{k}[14]$. The main drawback of $\Delta^{k}$ is its hypersensitivity to false positive points, i.e. this measure tends to over-penalize images with false detections. Indeed, when a false positive pixel is far from the true edge, the $\left|w\left(d_{G_{t}}(p)\right)-w\left(d_{D_{c}}(p)\right)\right|$ value creates a high impact on the evaluation, thus penalizing the measure (as in the example in Fig. 8).

Another way to compute a global measure is presented in [43] with the normalized edge map quality measure $D_{p}$. In fact, this distance measure is similar to $S F o M$, with different coefficients. The over-segmentation measure (left term) evaluates $d_{D_{c}}$, the distances between the FPs and $G_{t}$.
The under-segmentation measure (right term) computes the distances of the FNs between the closest correctly detected edge pixel, i.e. $G_{t} \cap D_{c}$. That means that $\mathrm{FNs}$ and their distances are not counted without the presence of $\mathrm{TP}(\mathrm{s})$, and $D_{p}$ is sensitive to displacements of edges. Moreover, both the left and the right terms are composed of a $\frac{1}{2}$ coefficient, so in the presence of only under- or over-segmentation, the score of $D_{p}$ does not go above $\frac{1}{2}$.

3) A new edge detection measure evaluation: In [37], a normalized measure is developed after computation of the edge assessment $\Gamma$. The $\Gamma$ function represents an over-segmentation measure which depends also of $F N$ and $F P$. As this measure is not sufficiently efficient concerning FNs, $\Psi$ is an alternative function which also considers $d_{D_{c}}$ for false negative points. Inspired by $S^{k}, \Psi$ uses different coefficients which change the behavior of the measure, as discussed in the next subsection.

\section{B. On the importance of the coefficients}

As shown in Table IV, distance measures compute the evaluation using a coefficient mostly including: $\left|G_{t}\right|,\left|D_{c}\right|$, $\left|G_{t} \cup D_{c}\right|, F N$ or $F P$. Concerning the $\Upsilon$ distance measure, the coefficient $\frac{100}{|I|}$ compress the measurement, especially when the image is large (as demonstrated in Fig. 12). For $D_{p}$, the coefficient $\frac{1}{|I|-\left|G_{t}\right|}$ affects the measure concerning the false positive term, thereby creating an insensitivity to FPs, because, generally, $\left|G_{t}\right| \ll|I|$ and $\frac{1}{|I|-\left|G_{t}\right|} \sim \frac{1}{|I|} \sim 0^{+}$. Thus, the assessment of FNs is given more weight in the edge evaluation, strongly penalizing edge displacements. The experiments presented in the next section illustrate this drawback (Fig. 12). However, $\Delta^{k}$ uses $|I|$ as denominator term because the mean distance is computed for all the pixels of the image.

The authors of $\Gamma$ have studied the influence of the coefficient in different concrete cases [37]. Actually, using only $\left|G_{t}\right|$ or $\left|D_{c}\right|$ penalizes severely the measurement when one misclassified pixel is placed at a significant distance of its true position.

TABLE IV

PARAMETERS AFFECTING THE ERROR DISTANCE MEASURES.

\begin{tabular}{|c|c|c|c|c|c|}
\hline Measure & $G_{t}$ & $\left|D_{c}\right|$ & $d_{G_{t}}$ & $d_{D_{c}}$ & Other \\
\hline FoM & $\checkmark$ & $\checkmark$ & $\checkmark$ & & \\
\hline $\mathrm{FoM}_{e}$ & & & $\checkmark$ & & $F P$ \\
\hline$F$ & $\checkmark$ & & & $\checkmark$ & $F P$ \\
\hline$d_{4}$ & $\checkmark$ & $\checkmark$ & & & $T P, F P, F N$ \\
\hline$M F o M$ & $\checkmark$ & $\checkmark$ & $\checkmark$ & $\checkmark$ & \\
\hline SFoM & $\checkmark$ & $\checkmark$ & $\checkmark$ & $\checkmark$ & \\
\hline$D_{p}$ & $\checkmark$ & & $\checkmark$ & & $|I|, p \in G_{t}: d_{G_{t} \cap D_{c}}(p)$ \\
\hline$\Upsilon$ & & & $\checkmark$ & & $|I|$ \\
\hline$H, H_{n \%}$ & & & $\checkmark$ & $\checkmark$ & \\
\hline$D^{k}$ & & $\checkmark$ & $\checkmark$ & & \\
\hline$\Theta$ & & & $\checkmark$ & & $F P$ \\
\hline$\Omega$ & & & & $\checkmark$ & $F N$ \\
\hline$\Delta^{k}$ & & & $\checkmark$ & $\checkmark$ & $|I|$ \\
\hline$f_{2} d_{6}$ & $\checkmark$ & $\checkmark$ & $\checkmark$ & $\checkmark$ & \\
\hline$S^{k}$ & & & $\checkmark$ & $\checkmark$ & $\left|G_{t} \cup D_{c}\right|$ \\
\hline$\Gamma$ & $\checkmark$ & & $\checkmark$ & & $F P+F N$ \\
\hline$\Psi$ & $\checkmark$ & & $\checkmark$ & $\checkmark$ & $F P+F N$ \\
\hline
\end{tabular}




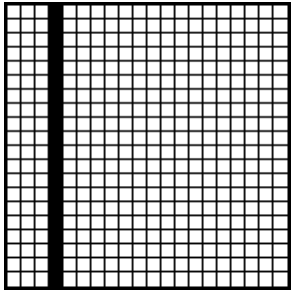

(a) Ground truth

$\left|G_{t}\right|=20$

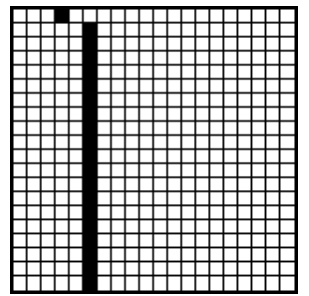

(b) $T_{1}$ with 19 FPs, distance of 2 pixels.

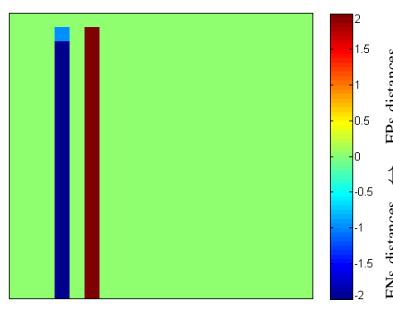

(c) Distance map from (a) vs. (b)

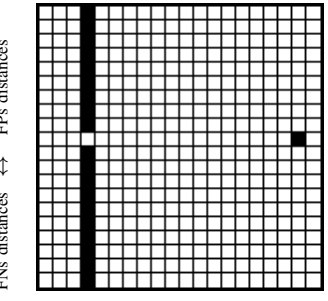

(d) $T_{2}$ with $1 \mathrm{FP}$, distance of 15 pixel.

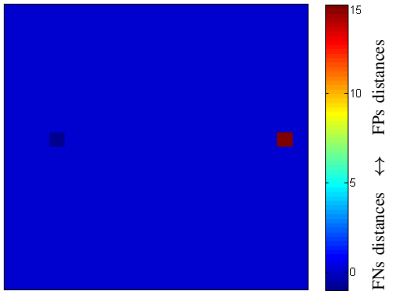

(e) Distance map from (a) vs. (d)

\begin{tabular}{|c|c|c|c|c||c|c|c|c|c|c|c|c|}
\hline$D_{c}$ & $\left|G_{t}\right|$ & $\sqrt{\sum_{p \in G_{t}} d_{D_{c}}^{2}(p)+\sum_{p \in D_{c}} d_{G_{t}}^{2}(p)}$ & $F N$ & $F P$ & $F o M_{e}$ & $H$ & $H_{20 \%}$ & $\Delta^{k}$ & $S^{k}$ & $\mathcal{A}$ & $\mathcal{B}$ & $\Psi$ \\
\hline \hline$T_{1}$ & 100 & 12.21 & 19 & 19 & 0.5 & 2 & 2 & 1.91 & 1.93 & 0.031 & 0.031 & 1.15 \\
\hline$T_{2}$ & 100 & 15.03 & 1 & 1 & 0.983 & 15 & 4 & 4.78 & 2.38 & 0.038 & 0.038 & 0.075 \\
\hline
\end{tabular}

Fig. 8. Errors quantified by $F_{o} M_{e}, H, H_{5 \%}, \Delta^{k}, S^{k}(k=2), \mathcal{A}, \mathcal{B}$ and $\Gamma$ for two different candidate edge images compared to the same ground truth.

For a counterexample, let us consider the following formula:

$$
\mathcal{A}\left(G_{t}, D_{c}\right)=\frac{\sqrt{\sum_{p \in G_{t}} d_{D_{c}}^{2}(p)+\sum_{p \in D_{c}} d_{G_{t}}^{2}(p)}}{\left|G_{t}\right|^{2}} .
$$

$\mathcal{A}$ represents an evaluation measure of contour detection involving both $d_{D_{c}}$ and $d_{G_{t}}$ plus a coefficient term $\frac{1}{\left|G_{t}\right|^{2}}$. Now, considering two images (images (b) and (d) in Fig. 8) with identical ground truth points $G_{t}$ (Fig. 8 a)). However, in image (d), a single FP point at a distance sufficiently high behaves for $\mathcal{A}$ as several FP points on another image; these images have the same interpretation in terms of $\mathcal{A}$ measure, which is not the case with ratios $1 / 10$ and $9 / 10$. The table in Fig. 8 illustrates, with a simple example, this drawback for $\mathcal{A}$, $\Delta^{k}$ and $S^{k}$, and a similar example can be found in in Fig. 7 .

Close to $\mathcal{A}$, the following measure $\mathcal{B}$ depends strongly on the desired contour number $\left|D_{c}\right|$ :

$$
\mathcal{B}\left(G_{t}, D_{c}\right)=\frac{\sqrt{\sum_{p \in G_{t}} d_{D_{c}}^{2}(p)+\sum_{p \in D_{c}} d_{G_{t}}^{2}(p)}}{\left|D_{c}\right|^{2}} .
$$

$\mathcal{B}$ suffers from the same problem as $\mathcal{A}$. Furthermore, in general, a measure depending mainly on a coefficient $\frac{1}{\left|D_{c}\right|}$ (as a coefficient $\frac{1}{|I|}$ ) evaluates a boundary image with a value close to 0 whereas $D_{c}$ stays totally misplaced, especially when $\left|D_{c}\right|$ is huge (when $\left|D_{c}\right|$ is huge, it corresponds to a total saturation of the desired contour with an inconsistent number of FPs).

Finally, the authors concluded in [37] that such a formulation must take into consideration all observable and theoretically observable cases, as illustrated in Fig. 8 That means that an effective measure has to take into account all the following input parameters $\left|G_{t}\right|,\left|D_{c}\right|, F N$ and $F P$, whereas the image dimensions should not be considered. Thus, the coefficient parameter $\frac{F P+F N}{\left|G_{t}\right|^{2}}$ seems a good compromise for an evaluation measure of edge maps and has been introduced into the new formula of assessment $\Psi$.

\section{Normalization of the edge detection evaluation}

In order to compare each boundary detection assessment, all the measures must be normalized, and must also indicate the same information: an error measure close to 1 means poor segmentation whereas a value close to 0 indicates good segmentation. The values of $F o M, F o M_{e}, F, d_{4}, M F o M$, $S F o M$ and $D_{p}$ comply with this $[0,1]$ condition. However, concerning the other distance measures in Table III normalization is required. Introduced in [37], a formula called Key Performance Indicator $(K P I)$, with $K P I \in[0,1]$ gives a value close to 1 for a poor segmentation. Alternatively, a $K P I$ value close to 0 indicates a good segmentation. The Key Performance Indicator is defined using the following equation:

$$
\begin{aligned}
K P I_{u}:[0 ; \infty[ & \mapsto[0 ; 1[ \\
u & \rightarrow 1-\frac{1}{1+u^{h}} .
\end{aligned}
$$

where the parameter $u$ is replaced by a distance error and $h$ a constant such that $h \in \mathbb{R}_{*}^{+}$.

A key parameter of the KPI formula is the power of the denominator term called $h$. It may be called a power of observation. Inasmuch as $K P I$ depends on its value, it evolves more or less quickly around 0.5 and embodies a range of observable cases. Average values have been determined for the error distance term $\sqrt{\sum d_{D_{c}}^{2}+\sum d_{G_{t}}^{2}}$ concerning $\Psi$ and $\sqrt{\sum d_{G_{t}}^{2}}$ for $\Gamma$. The choice of values between 1 and 2 can be easily checked. Otherwise, the more abrupt the KPI evolution, the less the transition between 0.5 and 1 is marked (i.e. the slope of the $K P I$ curve, for example $h=1$ in Fig. 97. Moreover, fixing $h=1, K P I$ stagnates far from 1 when $\sqrt{\sum d_{D_{c}}^{2}+\sum d_{G_{t}}^{2}}$ or $\sqrt{\sum d_{G_{t}}^{2}}$ becomes high. Additionally, when $h=2, K P I$ starts
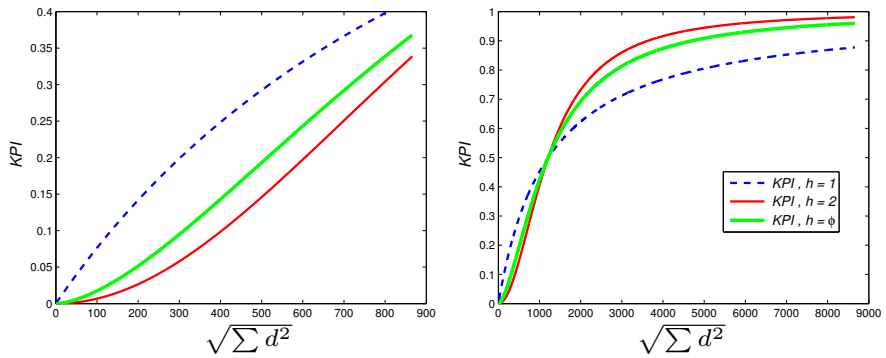

Fig. 9. Evolution of the $K P I_{\psi}$ in function of the mistake points distance, for different powers $h$, with $F N+F P=4000$ and $\left|G_{t}\right|=2200$. 

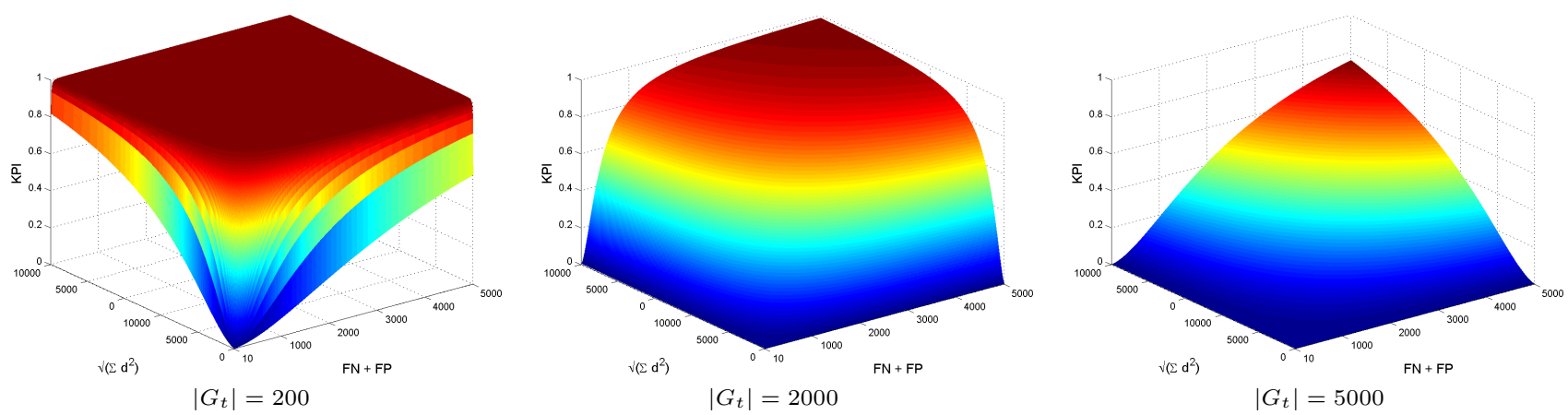

Fig. 10. Evolution of the KPI in function of the erroneous points distance, the number of FPs and FNs, for different $\left|G_{t}\right|$ and fixing $h=\phi$.

to increase slowly, then the slope becomes sharp around 0.5 and converges quickly towards 1 . Finally, to fix the power at the golden ratio $\phi \simeq 1.6180339887$ in order to ensure an evolution of $K P I$ that would not be too abrupt from 0 to 1 and also not penalize when the error distances are not high (contrary to $K P I$ with $h=1$, see Fig. 9). Furthermore, Fig. 10 illustrates $K P I$ values in function of the distance of the mistake points and their numbers. For a small skeleton $G_{t}=200$, it shows that $K P I$ draws near 1 quickly when the number of FPs, number of FNs and their distances are growing. Alternatively, for a bigger skeleton, $K P I$ rises more slowly. These three evolution surfaces show the coherence and robustness of $K P I$ regarding the starting conditions: a displaced edge is penalized in function of the number of false pixels and also of the distance from the position where it should be located.

Such a value enables a displaced edge to be penalized in function of the false pixel number and also of the distance from the position it should be located at. As a compromise, using the KPI formula with $h=\phi$, the measurement neither becomes too strong in the presence a small number of positive or negative misclassified pixels nor penalizes $D_{c}$ too severely if $d_{G_{t} / D_{c}}$ is small. Compared to $\Gamma, \Psi$ improves the measurement by combining both $d_{G_{t}}$ and $d_{D_{c}}$. The next section is dedicated to experimental results where candidate edge images are progressively degraded in order to study the behaviors of the presented boundary detection assessments. Note that the other measures are also normalized with another formula in the next section in order to compare all of them.

\section{COMPARISON OF EXPERIMENTAL RESULTS}

The two sections above describe the main error measures concerning edge detection assessment, explaining the advantages and drawbacks of each one. The tests carried out in the experiments are intended to be as complete as possible, and thus as close as possible to reality. To that end, considering an edge model (i.e. ground truth) the edge detection evaluation measures are subjected to the following studies:

- addition of false negative points (under-segmentation),

- addition of false positive points (over-segmentation),

- addition of both false negative and false positive points,

- addition of false positive points close to the true contour,

- translation of the boundary,
- remoteness of a false positive and false negative chains,

- computation of the minimum value of the measures on edge images (synthetic and real) compared to the ground truth.

Therefore, 26 measures are tested and compared with each other: statistical measures in Table $\mathrm{I}$ (except $B_{S N R}$ ), distance measures in Table II plus SFoM, MFoM and SSIM. Firstly, the normalized measures $\Phi^{*}, \chi^{2 *}, P_{m}^{*}, F_{\alpha}^{*}, S S I M$, $F o M, F o M_{e}, F, d_{4}, S F o M, M F o M, D_{p}, K P I_{\Gamma}$ and $K P I_{\Psi}$ are plotted together. The values of $S S R$ are not recorded because this measure behaves like $P_{m}^{*}$. Secondly, the scores of the other measures are also normalized to be plotted together, and normalized using the following equation for easy visual comparison. Denoting by $f \in[0 ;+\infty[$ the scores vector of a distance measure such that $m=\min (f)$ and $M=\max (f)$, then the normalization $\mathcal{N}$ of a measure is computed by:

$$
\mathcal{N}(f)=\left\{\begin{array}{cl}
0 & \text { if } M=m=0 \\
1 & \text { if } M=m \neq 0 \\
\frac{f-m}{M-m} & \text { if } M>1 \text { and } m \neq 0 \\
f & \text { otherwise. }
\end{array}\right.
$$

Note that the parameters for each evaluation measure are indicated directly in the captions of the curves and that the matlab code of the distance measures as FoM, $D^{k}, S^{k}$ and $\Delta^{k}$ are available at http://kermitimagetoolkit.net/library/code/

\section{A. Behavior comparison}

The simulation of the degradation of the ground truth $G_{t}$ is studied using synthetic data. Thus, concerning the first 6 experiments, a vertical line represents $G_{t}$ in a size image of $100 \times 100$, as illustrated in Fig 11 (a). FNs, FPs or displacements corrupt the image, and, as $G_{t}$ corresponds to a line, the interpretation of the results remains simpler. For example, adding a false pixel to $G_{t}$ or a translation of $G_{t}$ can easily be represented mathematically whereas, for a more complicated shape, the translation of $G_{t}$ can cross other pixels and an appropriate measure will not obtain a monotonic score. Hence, the degradations applied to the vertical lines are chosen in order to obtain a monotonic score for a complete measure. Then, the following two tests concern a ground truth which is a square followed by the computation of the minimal value of the edge detection evaluation measure in 


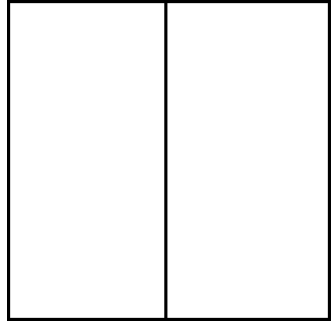

(a) $G_{t}$, image $100 \times 100$

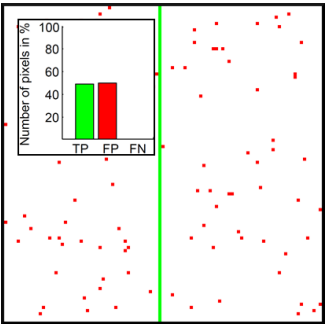

(b) addition of $101 \mathrm{FPs}$
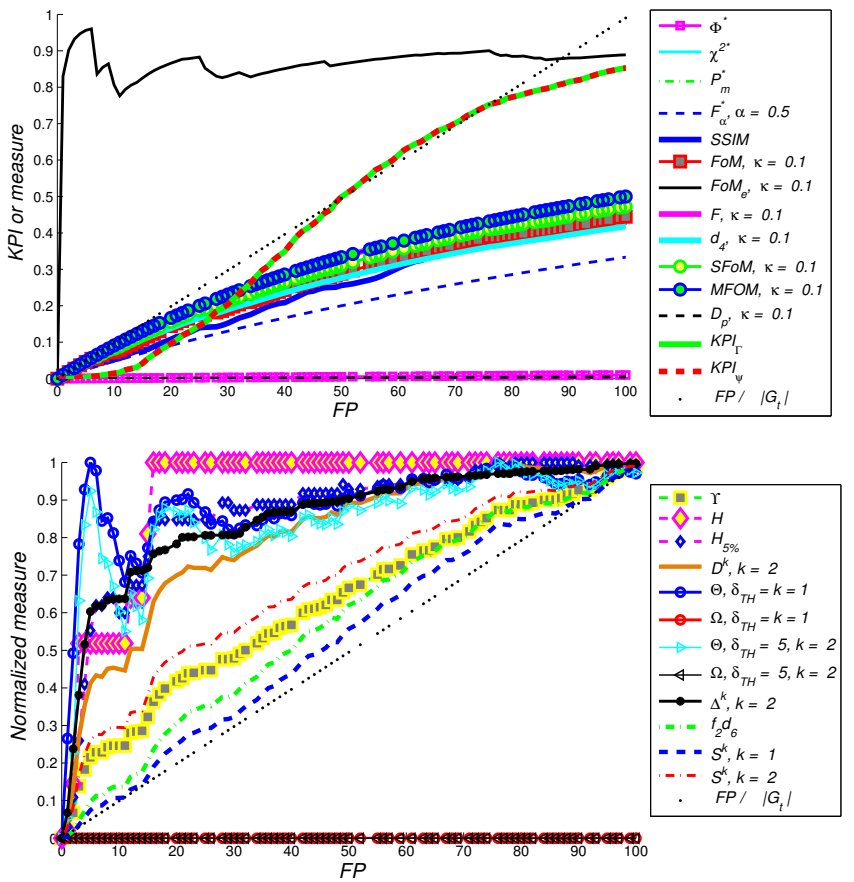

Fig. 11. Evolution of the dissimilarity measures in function of the number of added false positive points.

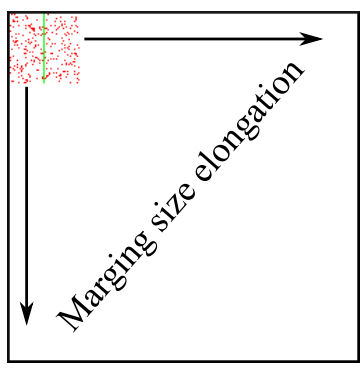

(a) Elongation of the margins

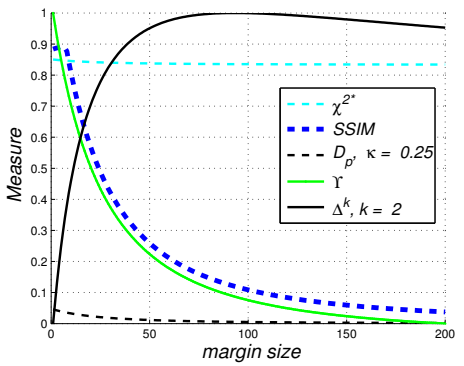

(b) Measure scores
Fig. 12. Behaviors of dissimilarity measures in function of image size.

function of the threshold.

1) Addition of false positive points: The first test consisted in randomly adding undesirable pixels to $G_{t}$ until 100 pixels, as represented in Fig. 11 (b). This perturbation is equivalent to impulsive noise. The curves in Fig. 11 indicate the response of each measure in function of the number of created Fs; they enable comparison of the results delivered by the different measures.

Firstly, concerning measures automatically given values between 0 and $1, \chi^{2 *}, P_{m}^{*}, F_{\alpha}^{*}, S S I M, F o M, F, d_{4}, S F o M$ and $M F o M$ overlap and penalize corrupted images by FPs as soon as they appear, but their values are under 0.5 whereas the last corrupted image is composed of more FPs that TPs(see Fig. 11 b)). FoM $M_{e}$ is not monotonic and computes a mean in function of the apparition of FPs. $\Phi^{*}$ and $D_{p}$ overlap and stay close to 0 (same remark for the under-segmentation $\Omega$ ). Contrary to the previous measures, $K P I_{\Gamma}$ and $K P I_{\Psi}$ ensure an evaluation which does not become too high in the presence of a small number of FPs, but penalizes $D_{c}$ more severely for 50 or more undesirable points. Secondly, concerning the non-normalized measures, $\Theta$ is not monotonic or sensitive to FP distances, as $H$ and $H_{5 \%}$ which stay blocked at the higher distances values, whatever the number of FPs. Furthermore, $\Upsilon$, $D^{k}, \Delta^{k}, f_{2} d_{6}$ and $S^{k}$ have a coherent behavior, even though $\Upsilon, D^{k}, \Delta^{k}$ and $S^{k}$ (with $k=2$ ) are sensitive to FPs at the beginning of the assessment.

This first experiment is produced by randomly adding FPs to the same $G_{t}$ image. The second test is presented in the image of Fig. 12 (a) which is composed of a vertical line and 100 FPs randomly placed around the line. The first image in this test image is as large as the image in Fig. 11 b). In a second step, the TPs and FPs are maintained in the same position and the margins are increased, as illustrated in Fig. 12 (b), influencing the evaluation in certain cases. The curves presented in Fig. 12 (b) show which dissimilarity measure

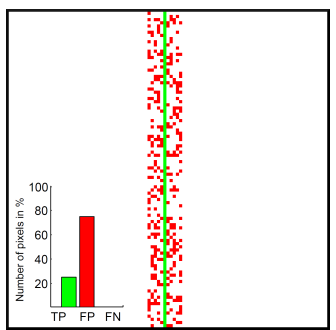

(a) $30 \%$ of FPs

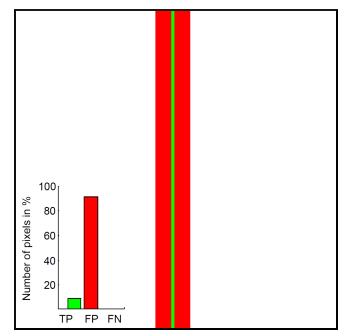

(b) $100 \%$ of FPs
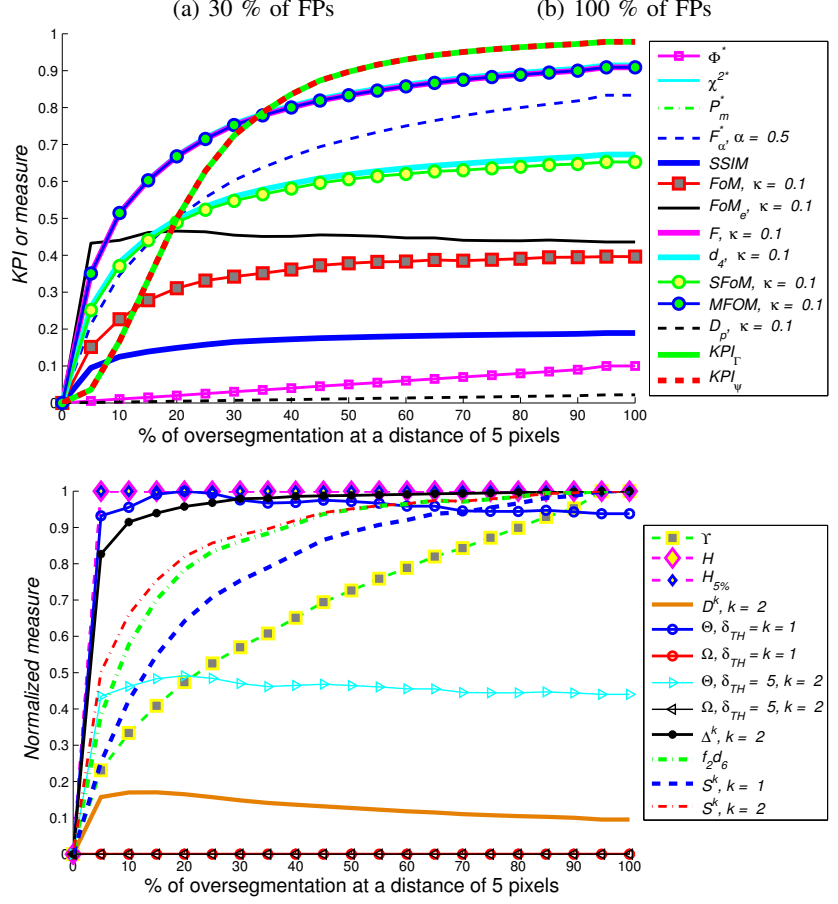

Fig. 13. Evolution of the dissimilarity measures in function of the oversegmentation in the contour area. 

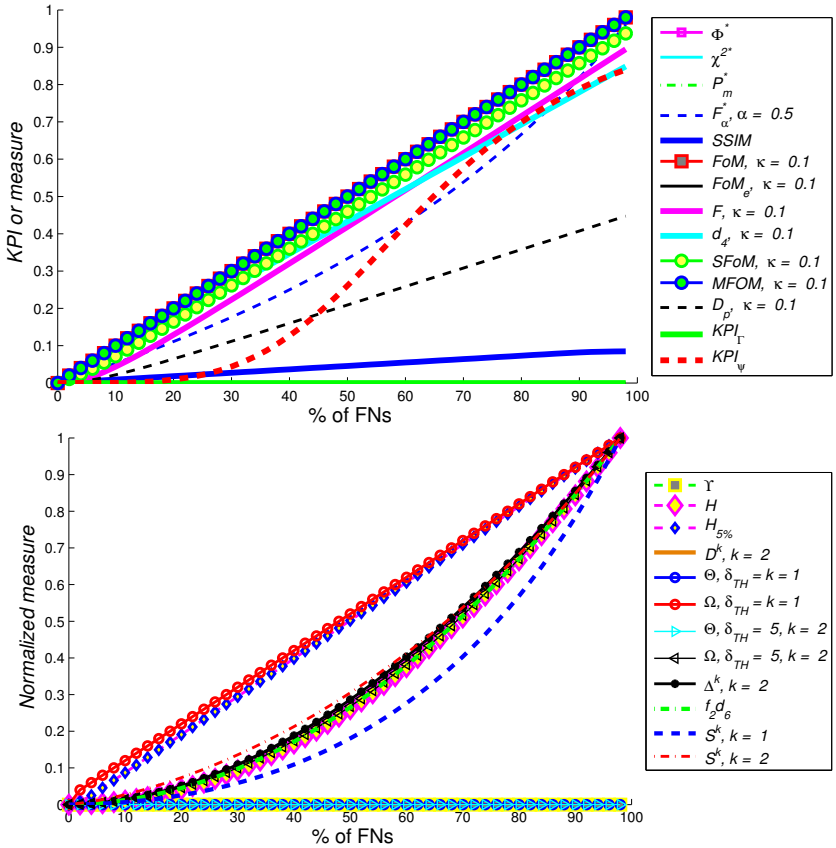

Fig. 14. Evolution of the dissimilarity measures in function of the number of false negative points addition. FoM and MFoM overlap.

scores are changed by the elongations of the margins (note that the margins lengthen both downward and to the right at the same time). Even though the values of $\chi^{2 *}$ do not change significantly, SSIM, $\Upsilon$ and $\Delta^{k}$ evolve strongly. The distance measure $D_{p}$, which remains insensitive to FPs becomes increasingly close to zero as the margin increases.

2) Over-segmentation close to the contour: The idea of this experiment is to create an over-segmentation at a maximum distance of 5 pixels, as illustrated in Fig. 13 a). Therefore, $100 \%$ of over-segmentation represents a dilation of the vertical line with a structural element of size $1 \times 6$, corresponding of a total saturation of the contour, see Fig. 13 b). The curves presented in Fig. 13 show that $F_{o} M_{e}, \Upsilon$, $S^{k}(k=2), \Delta^{k}$ and $\Theta\left(\delta_{T H}=1\right)$ are very sensitive to FPs whereas, $\Phi^{*}, D^{k}, F o M_{e}, S S I M$ and $D_{p}$ do not penalize $D_{c}$ enough. Also, $\Omega$ stagnates at 0 because it corresponds to an under-segmentation measure. $F o M_{e}, D^{k}$ and $\Theta$ are not monotonic whereas the saturation of the contour is progressive. Note that $H$ and $H_{5 \%}$ keep the same result throughout the test after $5 \%$ of degradation and that $\Delta^{k}$ is nearly the same. Finally, $\chi^{2 *}, F_{\alpha}^{*}, F_{o M}, F, d_{4}, S F_{o M}$, $M F o M, K P I_{\Gamma}, K P I_{\Psi}, f_{2} d_{6}$ and $S^{k}(k=1)$ ensure a measure evolution which is not too abrupt, even though FoM stagnates around 0.35, then $d_{4}$ and $S F O M$ around 0.6.

3) Addition of false negative points: In this experiment, pixels of the vertical line are missing, creating false negative points. Fig. 14 presents the evolution of the criteria obtained by the studied segmentation measures. These curves indicate that almost all the measures show the same behavior. The over-segmentation measures $F o M_{e}, \Gamma, \Upsilon, D^{k}$ and $\Theta$ stagnate at 0 . The SSIM stays close to 0 , whereas there are almost no more remaining pixels in $D_{c}$ at the end of
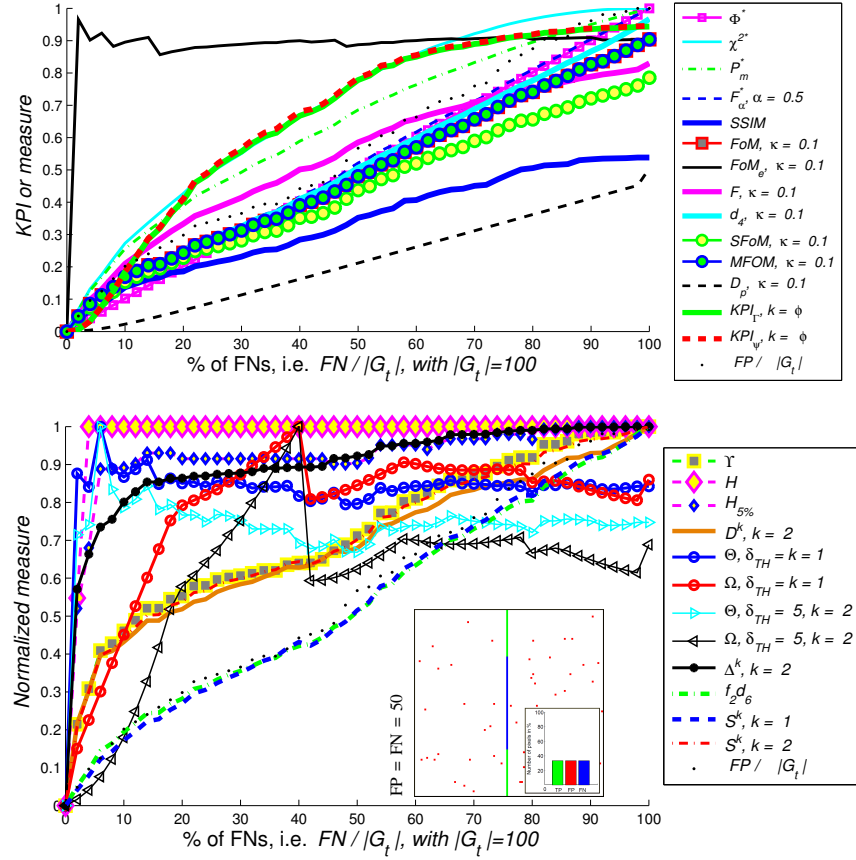

Fig. 15. Evolution of the dissimilarity measures in function of both the number of false positive and false negative points addition.

the experiment. As this test concerns only the addition of FNs, the $D_{p}$ distance measure obtains a result of 0.5 at the finish, but no higher because it corresponds to a measure which separates under- and over-segmentation. As feared in Section III the $d_{4}$ measure does not sufficiently penalizes the under-segmentation because the maximum score it attains is around 0.8. Moreover, compared to the experiment in Fig. 11] it is clear that FoM, F, $d_{4}, S F o M, M F o M$ and $D_{p}$ are more sensitive to $\mathrm{FN}$ addition than FP points. The
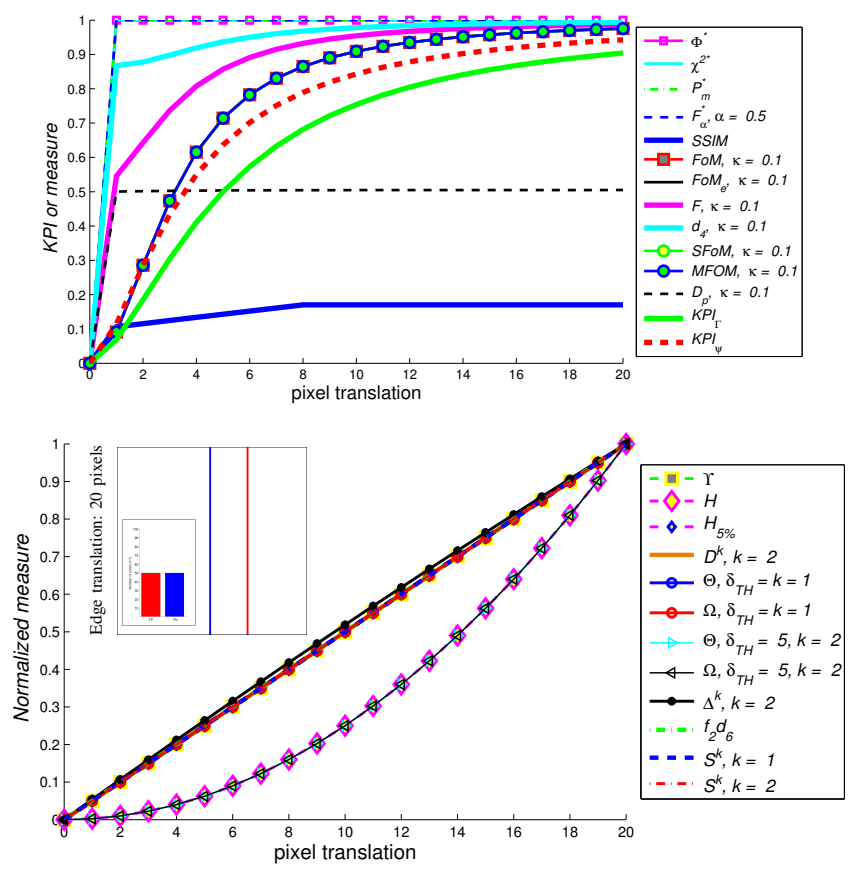

Fig. 16. Evolution of the dissimilarity measures in function of the translation of $D_{c}$. Note that $F o M, F o M_{e}, S F o M$ and $M F o M$ overlap. 


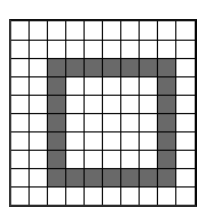

(a) $G_{t}$

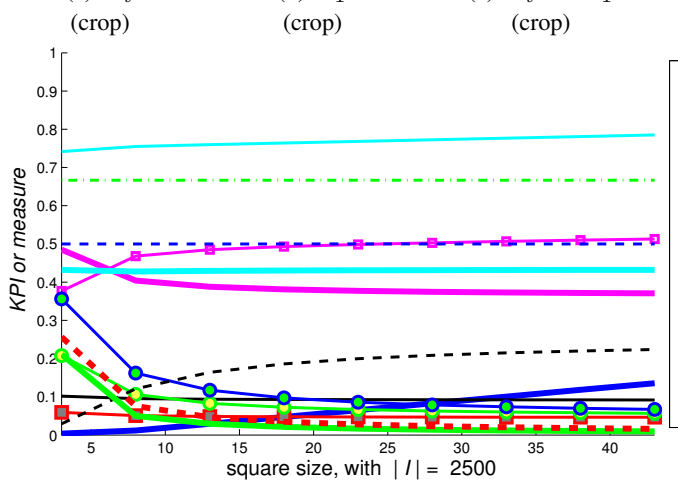

(i) $G_{t}$ vs. $C_{1}$ : Evolution of the normalized dissimilarity measures in function of the edges elongations

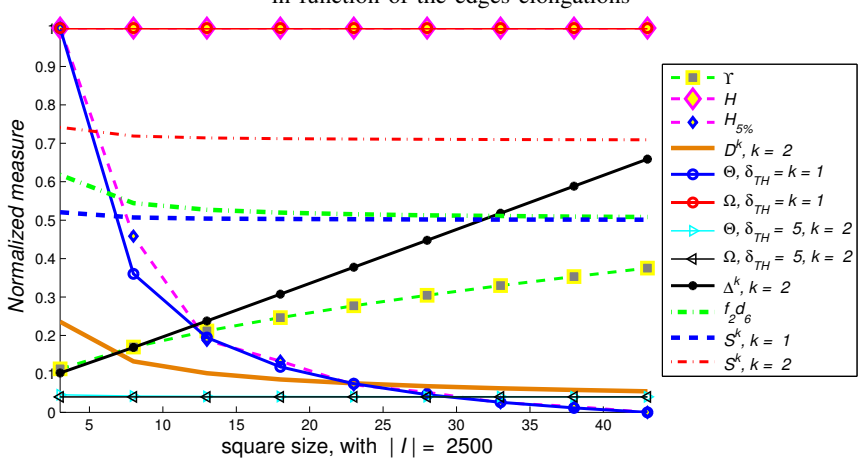

(k) $G_{t}$ vs. $C_{1}$ : Evolution of the dissimilarity measures in function of the edges elongations

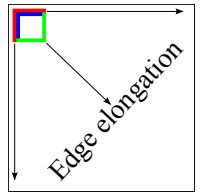

(d) Edges evolutions

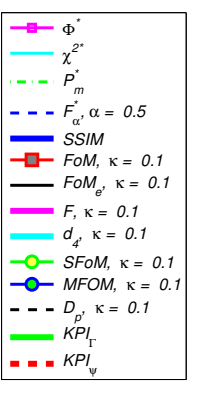
. 西

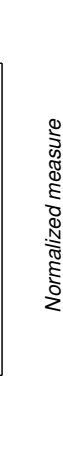 \\ :}

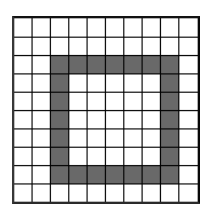

(e) $G_{t}$
(crop)

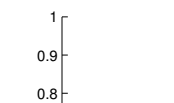

㲾 0.7

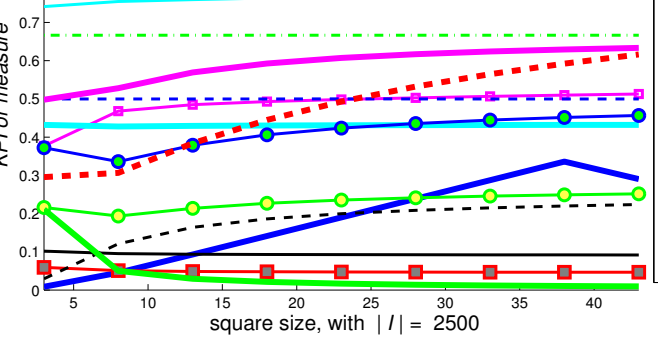

(j) $G_{t}$ vs. $C_{2}$ : Evolution of the normalized dissimilarity measures in function of the edges elongations

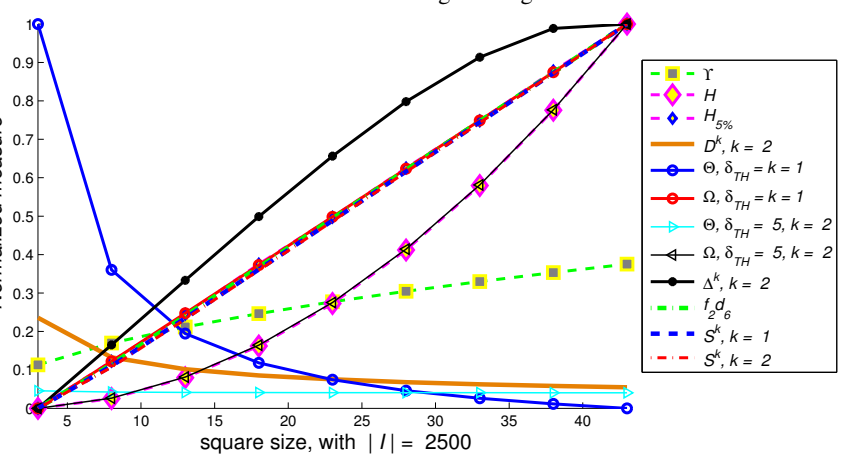

(l) $G_{t}$ vs. $C_{2}$ : Evolution of the dissimilarity measures in function of the edges elongations

Fig. 17. Evolution of dissimilarity measures in function of the size of the original square.

non-normalized measure scores in the lower graph of Fig. 14 evolve progressively, even though $S^{k}$ (with $k=1$ ) is less sensitive to FNs Note that, for $\Delta^{k}$ (which overlaps with $f_{2} d_{6}$ ), FN distances are considered to be less detrimental than FP distances (experiments reported in Fig. 11p. Finally, $K P I_{\Psi}$ ensures an evaluation which is not too high in the presence of a small number of FNs, followed by a greater penalization when the number of FNs increases.

4) Addition of both false positive and false negative points: Some measure are specialized and have an adaptive behavior for the evaluation of only under- or over-segmentation. Nevertheless, it is interesting to study their sensitivities by combining these two degradations because edge detectors often create both FPs and FNs in real/noisy images. Thus, adding randomly undesirable pixels to $G_{t}$ (until 100 pixels) and creating FNs at the same times gives a discontinuous line in the middle of randomly placed FPs, as illustrated in Fig 15 , bottom right.

Fig. 15 shows a hyper-sensitivity of FPs/FNs distances for $F o M_{e}, H, H_{5 \%}, \Theta, \Delta^{k}, S^{k}$ and $\Delta^{k}$. Moreover, $\Theta$ and $\Omega$ are not monotonic. Also, $\Phi^{*}, F_{\alpha}^{*}, F o M, d_{4}, M F o M, f_{2} d_{6}$ and $S^{k}$ (with $k=1$ ) increase (almost) constantly from 0 to
1 whereas both FNs and FPs are added. For example, the image in Fig 15 , bottom right, illustrates the line where both $50 \%$ of the correct pixels are missing and 50 FPs are added. In this precise case, $\Phi^{*}, F_{\alpha}^{*}, F o M, d_{4}, M F o M$ and $S F_{o M}$ measures give a result close to 0.5 whereas $G_{t}$ is disturbed by more than $50 \%$, as represented by the measurement of $\chi^{2 *}$, $P_{m}^{*}, K P I_{\Gamma}$ and $K P I_{\Psi}$ (note that $\Upsilon, D^{k}$ and $S^{k}$-with $k=2$ behave the same way even though they have a sensitivity to misplaced points at the beginning of the experiment). $F$ seems to evolve correctly but is not monotonic. Note that the SSIM remains unsuitable for this type of degradation because the best score it attains is little more than 0.5. Finally, the evolution of $D_{p}$ is monotonic until 0.5 but not thereafter, due to the coefficient $\frac{1}{|I|-\left|G_{t}\right|}$ which compresses the result, whereas the final image represents a cloud of FPs without TPs (the next experiment with edge translation shows more clearly the influence of this coefficient).

5) Edge translation: As pointed out in Section I a blur in the original image can shift the detected contour. When this displacement remains not too high, the shape of the desirable object stays detectable and the evaluation should not overly penalize the edge detector. Hence, edge detection evaluation 
measures must be assessed in function of the translation of a true contour. Indeed, the vertical line is shifted by a maximum distance of 20 pixels (Fig. 16 bottom left) and the score of the measures is plotted in Fig. 16 in function of the displacement of the desired contour. Thus, regarding the statistical measures $\Phi^{*}, \chi^{2 *}, P_{m}^{*}$ and $F_{\alpha}^{*}$, the curves perfectly illustrate the need to consider the distances of the misplaced pixels for the edge detection evaluation. FoM, $S F O M$ and $M F O M$ overlap completely, but their evaluations are correct, like $K P I_{\Gamma}$ and $K P I_{\Psi} . F$ and $d_{4}$ measures are sensitive to the first 2 translations. As pointed out above with the previous test, $D_{p}$ is not suited to the evaluation of translations, like $S S I M$ (the $S S I M$ computes locally a score in a window $8 \times 8$, so the score does not change after a switch of 8 pixels). The evolution of the other measures (Fig. 16. bottom) perform monotonically but no information can be infered because the measures are not normalized between 0 and 1 to evaluate the segmentation.

6) Comparison of the false positive distance and false negative distance points: In the experiment proposed in Fig. 17. two desired contours are compared with a ground truth, illustrating the importance of considering both the distance of the false negatives points $\left(d_{D_{c}}\right)$ and the distance of the false positive points $\left(d_{G_{t}}\right)$. Indeed, a square $\left(G_{t}\right.$ in Fig. 17 (a)) is compared with another square (Fig. 17 (b)) and also with a shape of two edges forming an angle (Fig. 17 (f)). The minimum square size for $G_{t}$ is $3 \times 3$ large and the image size stays fixed at $50 \times 50$. The shapes $C_{1}$ and $C_{2}$ keep the same position as in (c) and (g) and increase in proportion to $G_{t}$. All the shapes grow at the same time, as represented in Figs. 17 (d) and (h) and the scores for each measure are plotted in Fig. 17 (i), (j), (k) and (l). Thus, the more $G_{t}$ grows, the more $C_{1}$ is visually closer to $G_{t}$ whereas FNs deviate strongly in the case of $C_{2}$, but keep the same percentage of FPs and FNs for each desired contour. That is the reason why statistical measures obtain the same evolutions for each shape: $\Phi^{*}, \chi^{2 *}$, $P_{m}^{*}$ and $F_{\alpha}^{*}$. Despite these two different edge evolutions, some distance measures obtain almost the same measurements for $C_{1}$ and $C_{2}: F o M, F o M_{e}, D_{p}, D^{k}, \Theta$ (with $\delta_{T H}=1$ ) and $K P I_{\Gamma}$. On the contrary, concerning the normalized error distance measures, $M F o M$ and $K P I_{\Psi}$ increase to about 0.5 for $C_{2}$, due to around $50 \%$ of TPs whereas they converge towards 0 for $C_{1}$, since $C_{1}$ becomes visually closer to $G_{t}$. Nevertheless, $S F O M$ does not sufficiently penalize FN distances in $C_{2}$, and $F$ is too severe with $C_{1}$. Concerning non-normalized measures, $H_{5 \%}, f_{2} d_{6}$ and $S^{k}$ behave correctly.

\section{B. Threshold corresponding to the minimum measure}

The aim of the experiments presented here is to obtain the best edge map in a supervised way. The edges are extracted using the Canny filter [8] (i.e. isotropic Gaussian filter with a standard deviation of $\sigma$ ). Then, thin edges are created after the non-maximum suppression of the absolute gradient in the gradient direction $\eta$ [49] (see table V] and are normalized for easier comparison. In order to study the performance of the contour detection evaluation measures, one approach is to compare each measure by varying the threshold of the thin edges computed by an edge detector ${ }^{1}$ Indeed, compared to a ground truth contour map, the ideal edge map for a measure corresponds to the desired contour at which the evaluation obtains the minimum score for the considered measure among the thresholded gradient images. Theoretically, this score corresponds to the threshold at which the edge detection represents the best edge map, compared to the ground truth contour map [14][9]. Since a small threshold leads to heavy over-segmentation and a strong threshold may create numerous false negative pixels, the minimum score of an edge detection evaluation should be a compromise between under- and over-segmentation.

The two images used in these experiments are a synthetic and a real image. For the first one, in Fig. 18 (a), several white shapes are present immersed in a black background, creating step edges. To avoid the problem of edge pixel placements, as stated in Section [1. Fig. 1. a blur is created by adding a 1 pixel width of gray around each shape. Thus, the ground truth corresponds to this gray. The second image in Fig. 19.a) is a real image with a corresponding hand-made ground truth. Even though the problem of hand-made ground truths on real images is discussed by some researchers, only the comparison of $D_{c}$ with a $G_{t}$ is studied here.

1) Edges of synthetic data: To evaluate the measures performances, the original image in Fig. 18(a) is disturbed with random Gaussian noise and edges are extracted from the noisy image (Fig. 18(d) and (e)). Scores are plotted in function of the threshold and the image under each curve corresponds to the ideal edge map for the considerate measure. Statistic measures and $D_{p}$ correctly extract the edges at the price of numerous FPs. The Hausdorff distance $H, H_{5 \%}$ and $\Delta^{k}$ threshold the edges too severely, losing the majority of TPs. The over-segmentation distance measures $D^{k}, \theta$ and $\Gamma$ lose almost all the contours because the minimum corresponds to the threshold where no false pixel appears (even though true pixels disappear). Finally, $S S I M, f_{2} d_{6}$, $F, S^{k}$ and $\Psi$ do not create significant holes in the contours and the FNs extracted are positioned close to the true contours.

2) Edges of a real image: Real images contain other disturbances than the previous noisy synthetic image, such as texture or blurred edges. The ground truth and the original image in Fig. 19 arise from the database available at the following website: http://www.cs.rug.nl/ imaging/databases/ contour database/contour database.html

In edge detection assessment, the ground truth is considered as a perfect segmentation. However, the boundary benchmarks are built by human observers and errors may be created by human labels (oversights or supplements). Indeed, an inaccurate ground truth contour map in terms of localization (sometimes several pixels, see [27], part 2.2.2.3) penalizes precise edge detectors and/or advantages the rough algorithms. In [23] the

\footnotetext{
${ }^{1}$ The matlab code and several measures are available on MathWorks: https://fr.mathworks.com/matlabcentral/fileexchange/
} 63326-objective-supervised-edge-detection-evaluation-by-varying-thresholds-of-the-thin-ed 


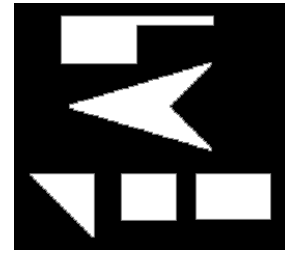

(a) Image $270 \times 238$

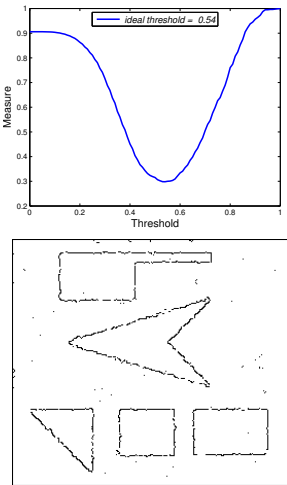

(g) $F_{\alpha}$
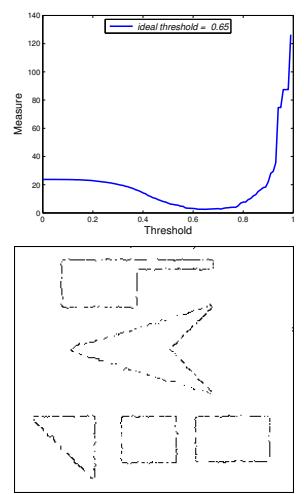

(m) $S^{k}, k=2$

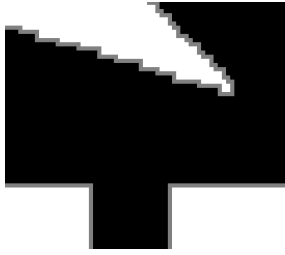

(b) Zoom in (a)
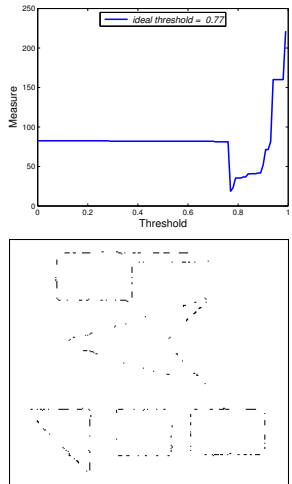

(h) $H$
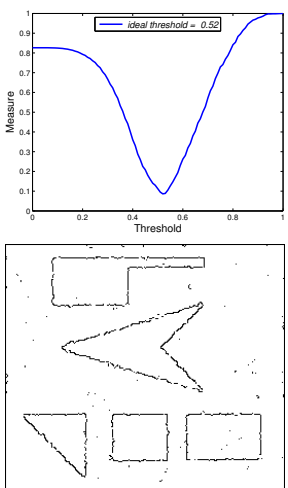

(n) $F o M$

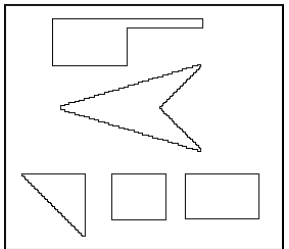

(c) $G_{t},\left|G_{t}\right|=1584$
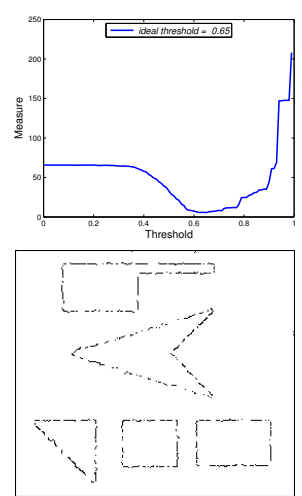

(i) $H_{5 \%}$
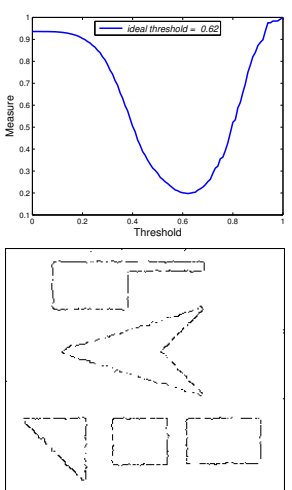

(o) $F$

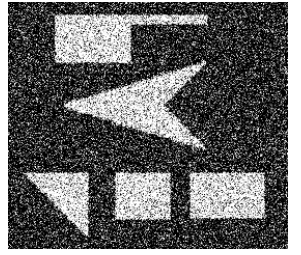

(d) Noisy, $S N R=3.31 \mathrm{~dB}$
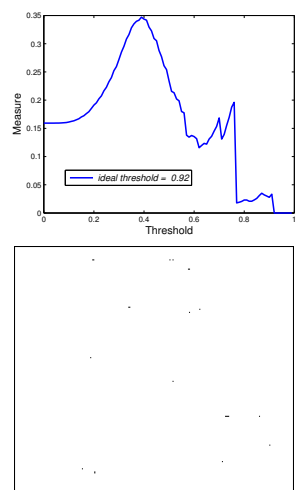

(j) $D^{k}, k=2$
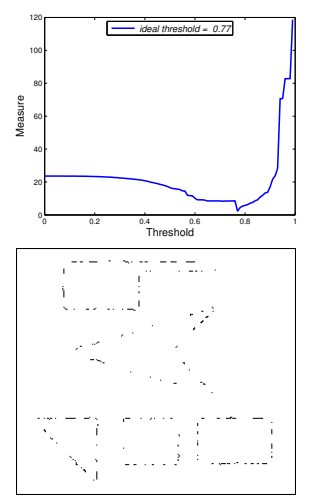

(p) $\Delta^{k}$

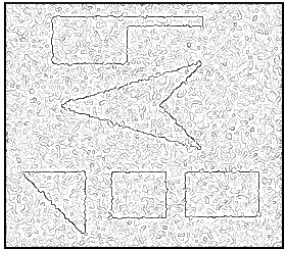

(e) Thin edges

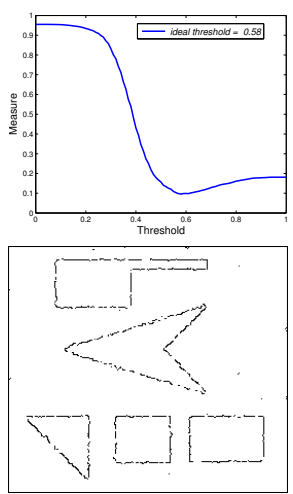

(k) $S S I M$
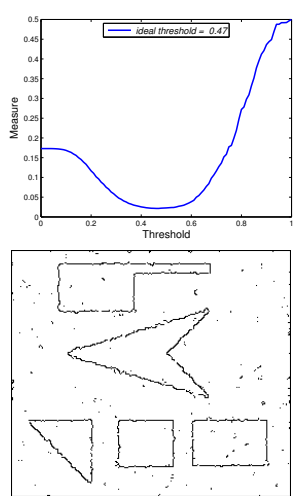

(q) $D_{p}$

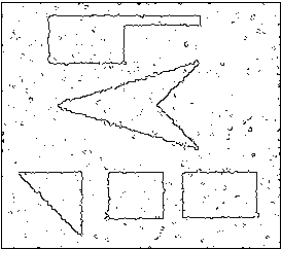

(f) $\Phi$
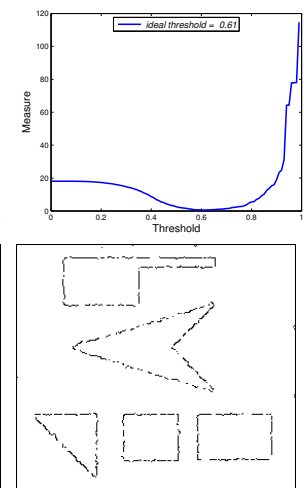

(l) $f_{2} d_{6}$
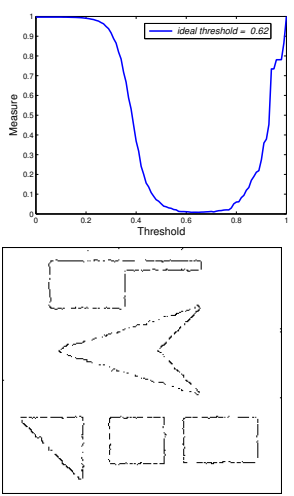

(r) $K P I_{\Psi}$

Fig. 18. Comparison of the minimal score for several edge detection evaluation measures concerning synthetic data. The edges are extracted using Canny's theory $(\sigma=1)$ on the noisy image in (d) and the binary images correspond to the thin edge image in (e) which is thresholded at the threshold corresponding to the minimum value of the bottom curve. The results for $F_{\alpha}^{*}$ are the same as the $P_{m}^{*}, \chi^{2 *}$ measures and do not need to be reported. The scores of the over-segmentation measure $D^{k}$ are the same as $F o M_{e}, \Upsilon, \Theta$ and $\Gamma$. Moreover, FoM, $d_{4}, S F o M$ and $M F o M$ obtain almost the same result.

question is raised concerning the reliability of the datasets regarded as ground truths for novel edge detection methods. Thus, an incomplete ground truth penalizes a method detecting true boundaries and efficient edge detection algorithms obtain between $30 \%$ and $40 \%$ of errors. Furthermore, contrary to the previous experiment, where only noise is added to the synthetic image, the true contours are only reported by human perception and, locally, the image intensities (for example the texture) create some disturbances which are nether spatially positioned as a result of the filtering technique, nor humanly perceptible. Unfortunately, this type of contour can be amplified by a strong gradient created by the edge detector [8]. As these undesirable pixels could be far from $G_{t}$, however, an edge detection method must converge to a threshold which preserves acceptable contours, close to the ground truth, and removes undesirable contour pixels. In other words, the final shapes created by the contours of the binarized image should be similar, especially nearby the edges pixels of $G_{t}$. Thus, in this case, the segmented images are totally different from $G_{t}$ than those in the synthetic case. For example, the ideal edges indicated by the $S S I M$ miss most of the main edges. Also, $P_{m}^{*}, F_{\alpha}^{*}, \chi^{2 *}$ and $d_{4}$ indicate an ideal edge image composed of numerous false positive pixels ( $d_{4}$ obtains this result because most statistics include this measure). On the contrary, the statistical measure $\Phi$, and the distance measures $H, D_{p}$ and $\Delta^{k}$ lose most of the desired boundaries; furthermore, the ideal threshold for $F o M_{e}$ and $\Upsilon$ is 1 . Ideal contour images concerning $S_{k=2}^{k}, H_{5 \%}, f_{2} d_{6}, F o M, S F_{o M}$ and $M F o M$ are less polluted by FPs. Finally, the ideal contour map computed for $\Psi$ is less noisy and better-quality, leading to a compromise between optimum under- and over-segmentation.

3) Comparison of several edge detectors by filtering: In this section, we compare the score of different evaluation measures involving real images (Figs. 20 b) and 26(a)) and 9 filtering edge detectors: Sobel [53], Shen [50], Bourennane [6], Deriche [11], Canny [8], Steerable filter of order $1\left(S F_{1}\right)$ [15], Steerable filter of order $5\left(S_{5}\right)$ [25], Anisotropic Gaussian Kernels (AGK) [16], Half Gaussian Kernels (H-K) [35]. Table 


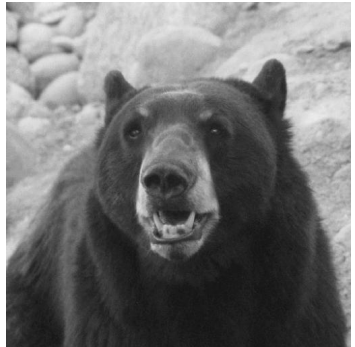

(a) Image $512 \times 512$

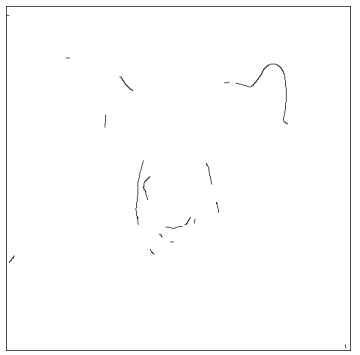

(h) $S S I M, D_{k=2}^{k}, t h=0.57$

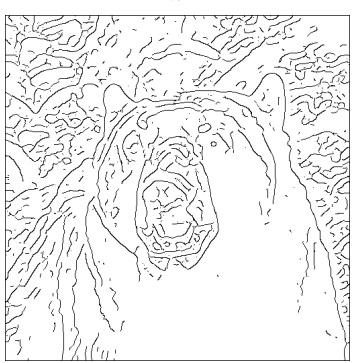

(m) $\Delta^{k}, t h=0.07$

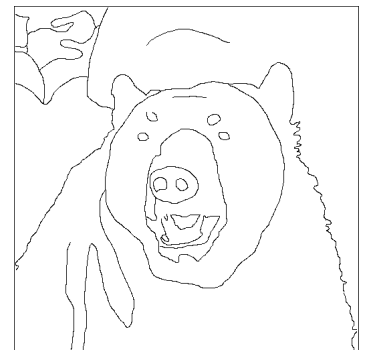

(b) $G_{t},\left|G_{t}\right|=5951$

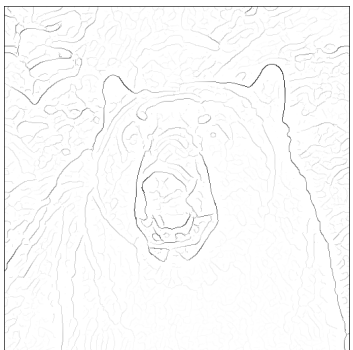

(c) Thin edges, $\sigma=3$

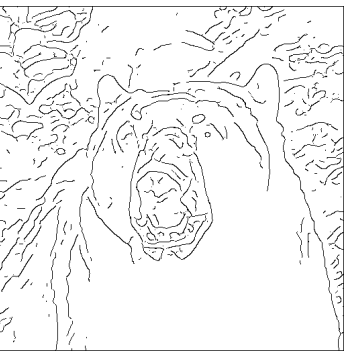

(i) $H, t h=0.1$

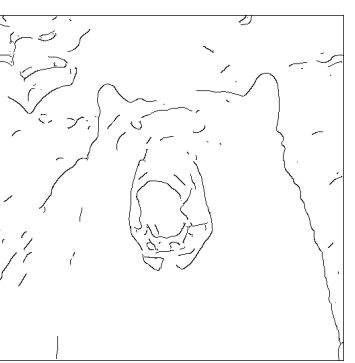

(n) $\Psi, t h=0.23$

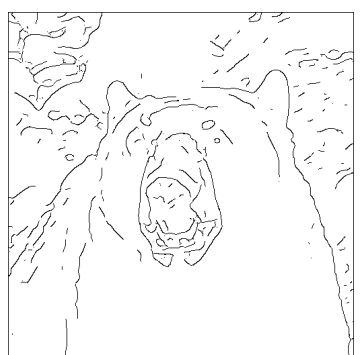

(j) $H_{5 \%}, t h=0.16$

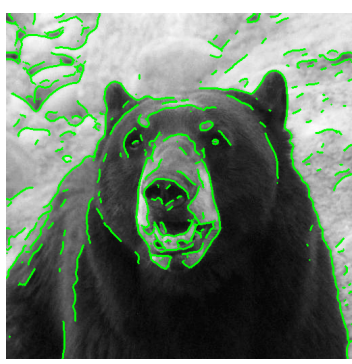

(o) Detected edges of ( $\mathrm{j}$ )

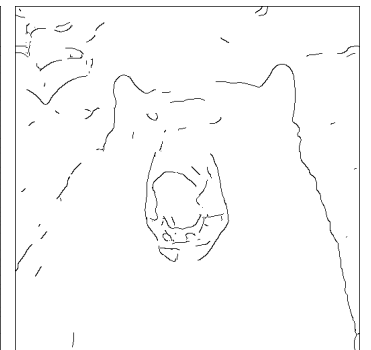

(d) $P_{m}^{*}, F_{\alpha}, \chi^{2 *}, d_{4} t h=0.27$

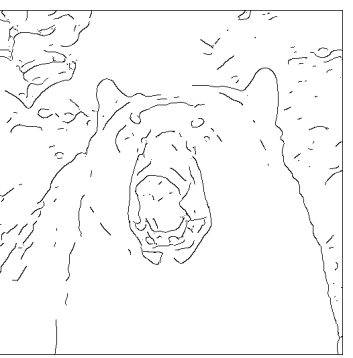

(k) $f_{2} d_{6}, S_{k=1}^{k}, F, t h=0.18$

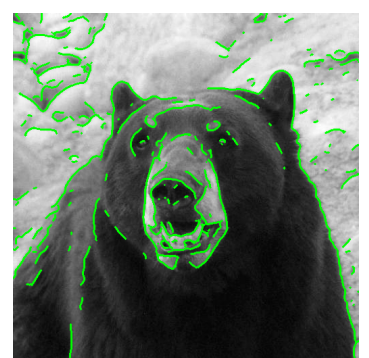

(p) Detected edges of (m)

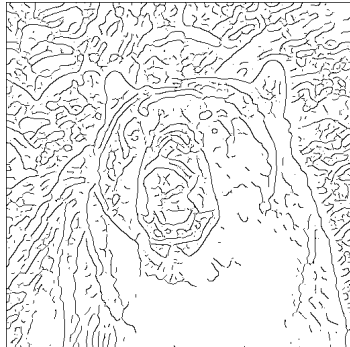

(e) $\Phi, D_{p}, t h=0.04$

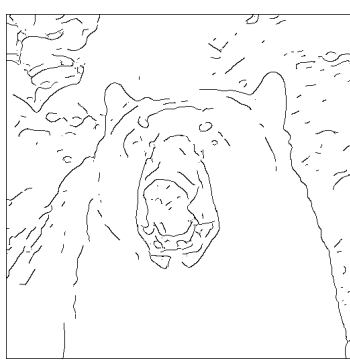

(1) $S_{k=2}^{k}, F o M, t h=0.17$

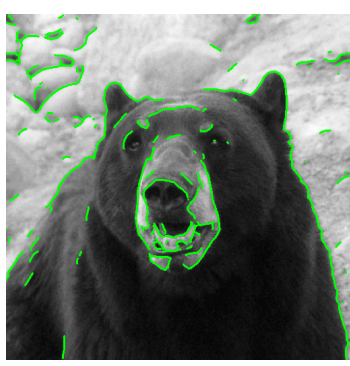

(q) Detected edges of (n)

Fig. 19. Comparison of the minimal score for several edge detection evaluation measures concerning a real image and a hand-made ground truth. The edges are extracted using Canny's theory $(\sigma=3)$ on the real image in (a) and the binary images correspond to the thin edge image in (c) which is thresholded at the value $(t h)$ corresponding to the minimum score for each measure. The best images for the measures $S_{k=1}^{k}, f_{2} d_{6}, S F o M$ and $M F o M$ are the same. The optimal threshold for the over-segmentation measures $\Theta_{\delta_{T H}=1 \text { or } 5}, \Gamma$ is $t h=1$, and is $t h=0$ for the under-segmentation measure $\Omega_{\delta_{T H}}=1$ or 5 .

V] shows how gradients are computed using these methods. The parameters of the filters are chosen to keep the same spatial support for the derivative information (see Fig. 27(o)). As above, the comparison is the same: the segmented image corresponds to the one having the minimum score for the considered measure.

Box [53] and exponential [50] [6] filters do not delocalize contour points [28] whereas they are sensitive to noise (i.e., addition of FPs). The Deriche [11] and Gaussian filters [8] are less sensitive to noise but suffer from rounding corners and junctions (see [28]) as the oriented filters $S F_{1}$ [15], $S F_{5}$ [25] and AGK [16], but the more the 2D filter is elongated, the more the segmentation remains robust against noise. Finally, as a compromise, $\mathrm{H}-\mathrm{K}$ correctly detects contours points having corners and is robust agains noise [35]. Consequently, the scores of the evaluation measures for the first 3 filters must be higher than the three last ones. Furthermore, the ideal segmented image should be visually closer to the ground truth edge image concerning $S F_{5}, \mathrm{AGK}$ and H-K. Thus, Fig. 21 illustrates that $F_{\alpha}$ gives coherent segmentation and scores even though it is high noisy for images (a)-(f) but the scores are not consistent for the noisy image in Fig. 26. a), see Fig. 27 a). $H$ and $\Delta^{k}$ measures are very sensitive to FPs, therefore, important contours are missing in the segmented images (Figs.
22 and 24). Worse still, concerning the image in Fig. 26.a), the scores of AGK are qualified as the second worst segmentation. The segmented images with respect to FoM remain noisy (Fig. 23) and the scores in Fig. 27.h) penalize both AGK and $\mathrm{H}-\mathrm{K}$ filters almost like the Sobel filter.

Finally, segmented images using $f_{2} d_{6}, S^{k}$ and $\Psi$ are close to the ground truth edge image and, the more efficient the edge detection filter is, the more the main contours are visible with reasonable FPs and the scores decrease in function of the effectiveness of the filter used. On the contrary, other evaluation measures give either segmented images with high level of FPs, or incoherent scores (bars in Figs. 20 and 27).

TABLE V

GRADIENT MAGNITUDE AND ORIENTATION COMPUTATION FOR A SCALAR IMAGE $I$ WHERE $I_{\theta}$ REPRESENTS THE IMAGE DERIVATIVE USING A FIRST-ORDER FILTER AT THE $\theta$ ORIENTATION (IN RADIANS).

\begin{tabular}{|l|c|c|}
\hline Type of operator & Gradient magnitude & Gradient direction \\
\hline $\begin{array}{l}\text { Fixed operator } \\
\text { [53], 50], 6], } \\
\text { 11], 8] }\end{array}$ & $|\nabla I|=\sqrt{I_{0}^{2}+I_{\pi / 2}^{2}}$ & $\eta=\arctan \left(\frac{I_{\pi / 2}}{I_{0}}\right)$ \\
\hline $\begin{array}{l}\text { Oriented Filters } \\
\text { 15], 25], 16] }\end{array}$ & $|\nabla I|=\max _{\theta \in[0, \pi[}\left|I_{\theta}\right|$ & $\eta=\underset{\theta \in[0, \pi[}{\arg \max }\left|I_{\theta}\right|+\frac{\pi}{2}$ \\
\hline $\begin{array}{l}\text { Half Gaussian } \\
\text { Kernels [35] }\end{array}$ & $|\nabla I|=\max _{\theta \in[0,2 \pi[} I_{\theta}-\underset{\theta \in[0,2 \pi[}{\min } I_{\theta}$ & $\eta=\left(\underset{\theta \in[0,2 \pi[}{\arg \max } I_{\theta}+\underset{\theta \in[0,2 \pi[}{\arg \min } I_{\theta}\right) / 2$ \\
\hline
\end{tabular}




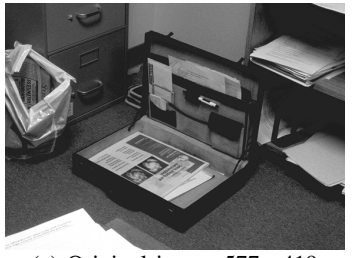

(a) Original image $577 \times 419$

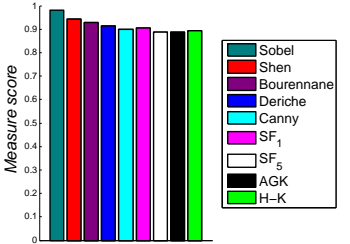

(f) $\chi_{2}^{*}$

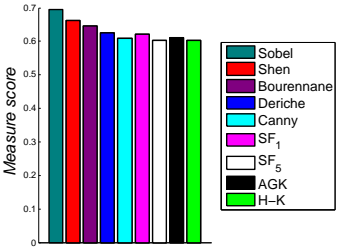

(k) $d_{4}$

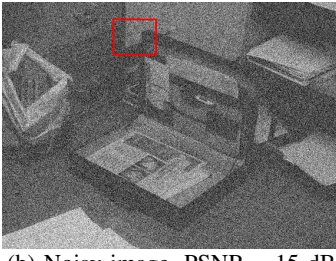

(b) Noisy image, PSNR $=15 \mathrm{~dB}$

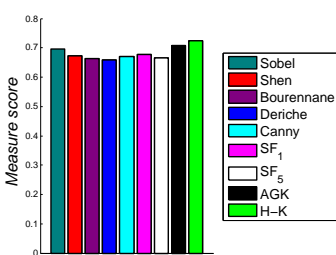

(g) $\Phi^{*}$

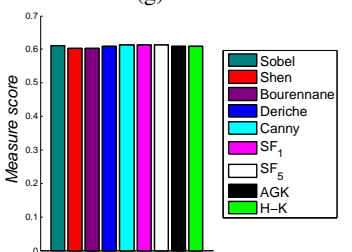

(l) $M F o M$

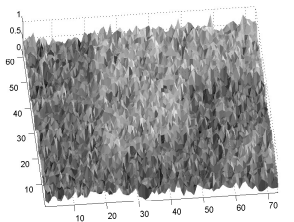

(c) Image surface in the square

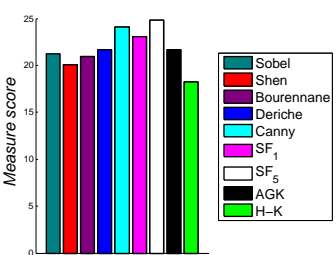

(h) $H_{5 \%}$

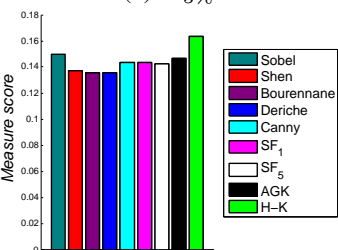

(m) $D_{p}$

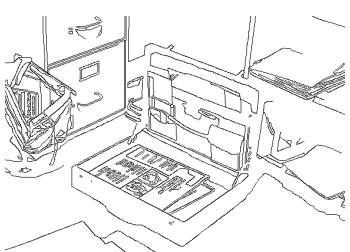

(d) Ground truth edge map

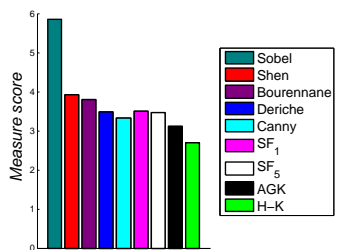

(i) $f_{2} d_{6}$

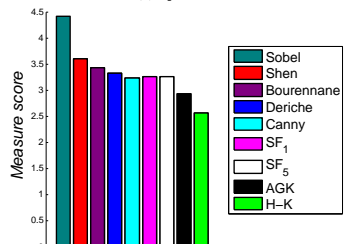

(n) $S_{k=1}^{k}$

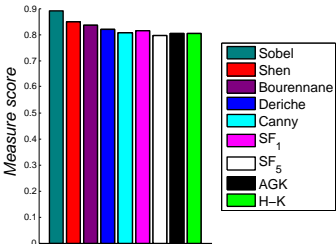

(e) $P_{m}^{*}$
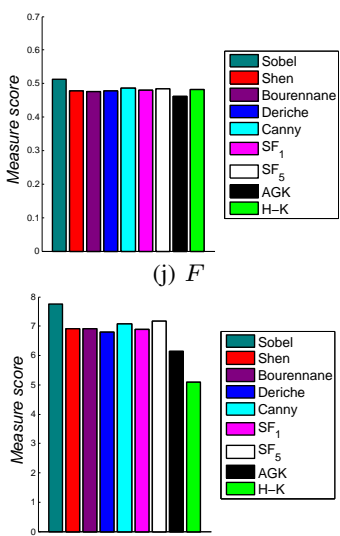

(o) $S_{k=2}^{k}$

Fig. 20. Comparison of the minimal score for several edge detection evaluation measures concerning a real image and several edge detection methods.

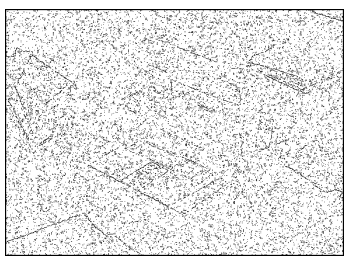

(a) Sobel

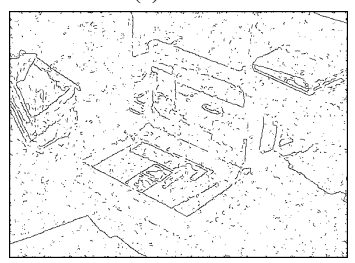

(f) $S F_{1}$

(g) $S F_{5}$
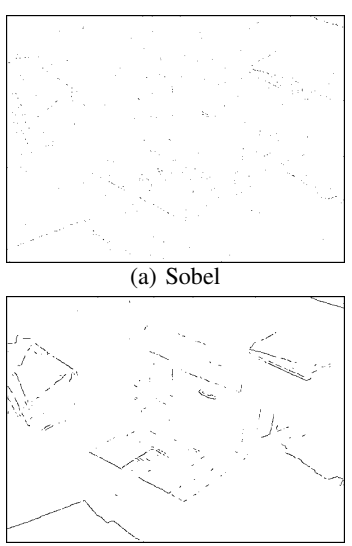

(f) $S F_{1}$

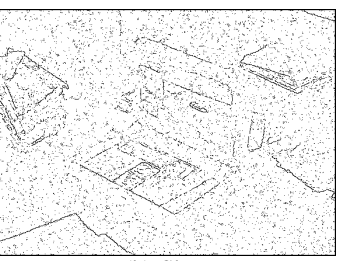

(b) Shen
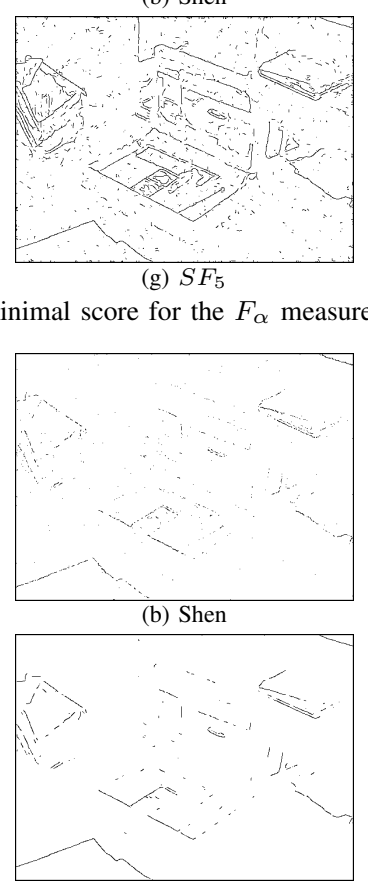

(g) $S F_{5}$

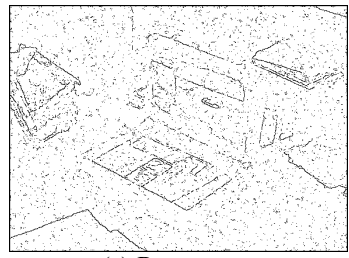

(c) Bourennane

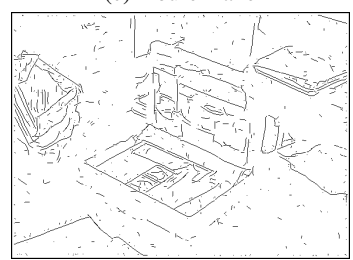

(h) AGK

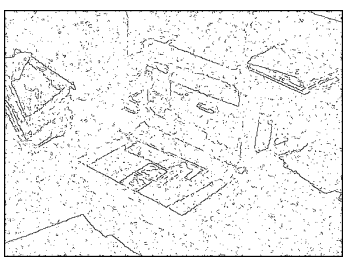

(d) Deriche

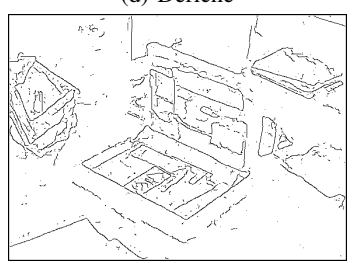

(i) $\mathrm{H}-\mathrm{K}$

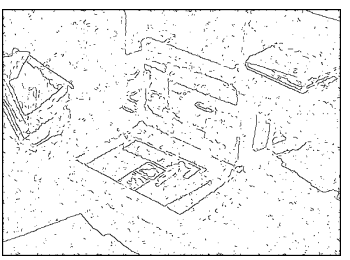

(e) Canny

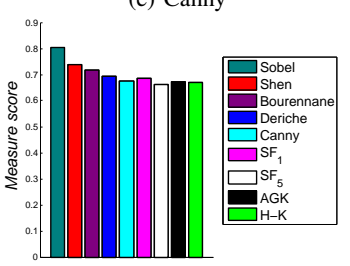

(j) Minimum scores of $F_{\alpha}^{*}$

Fig. 22. Comparison of the minimal score for the $H$ measure concerning a real image and several edge detection methods.

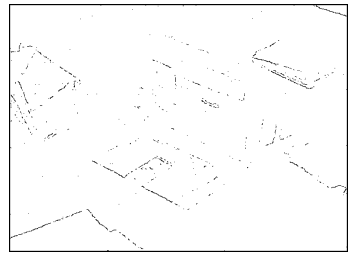

(c) Bourennane

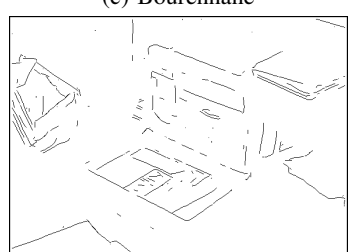

(h) AGK
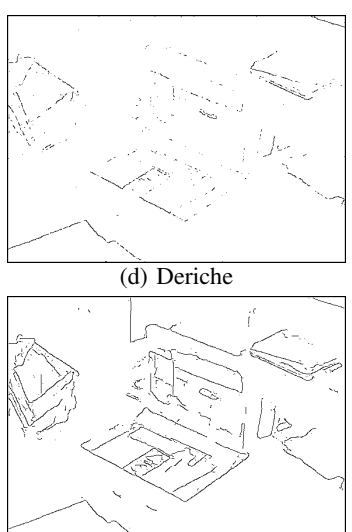

(i) $\mathrm{H}-\mathrm{K}$
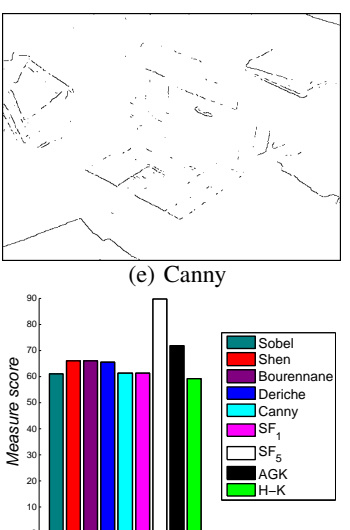

(j) Minimum scores of $H$

\section{CONCLUSION}

This study presents a review of supervised edge detection assessment methods. A supervised evaluation process estimates scores between two binary images: a ground truth and a candidate edge map. Eight statistical evaluations based on the number of false positive, false negative, true positive or true negative points are detailed. Firstly, examples of the evaluation of contour images prove the need to evaluate an edge detector using assessments involving distance measures. Fifteen distance measures are therefore also evaluated. The 

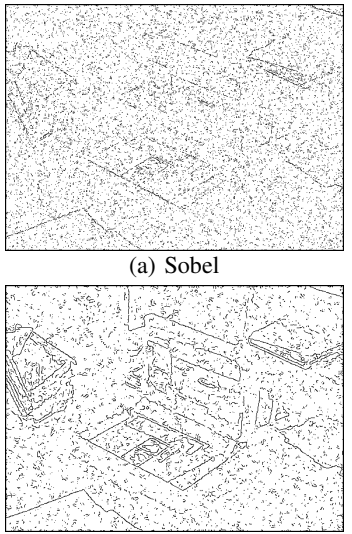

(f) $S F_{1}$

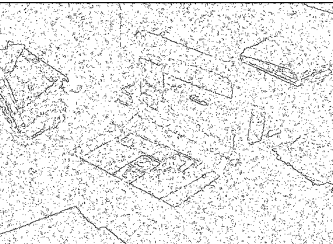

(b) Shen

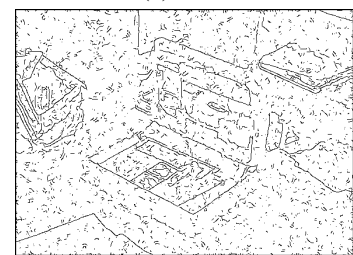

(g) $S F_{5}$

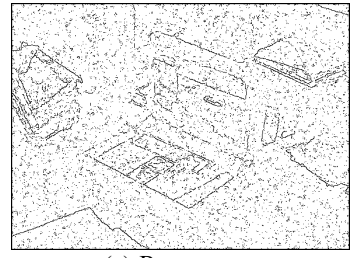

(c) Bourennane

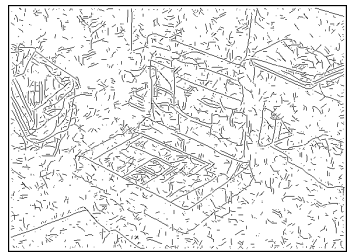

(h) AGK
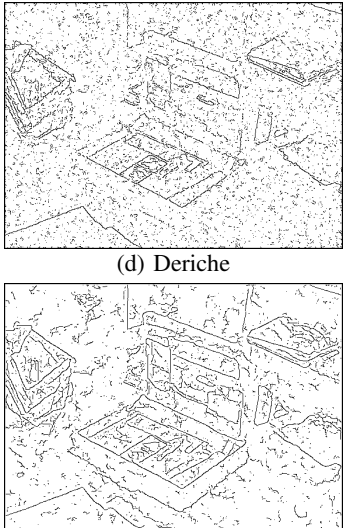

(i) $\mathrm{H}-\mathrm{K}$

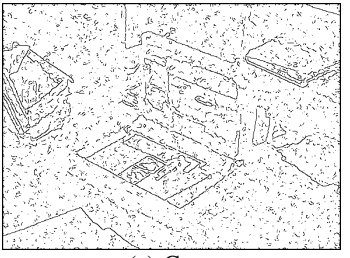

(e) Canny

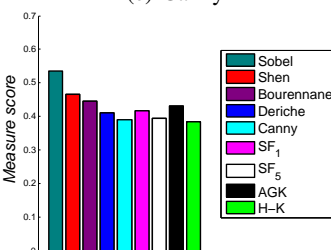

(j) Minimum scores of FoM

Fig. 23. Comparison of the minimal score for the FoM measure concerning a real image and several edge detection methods.
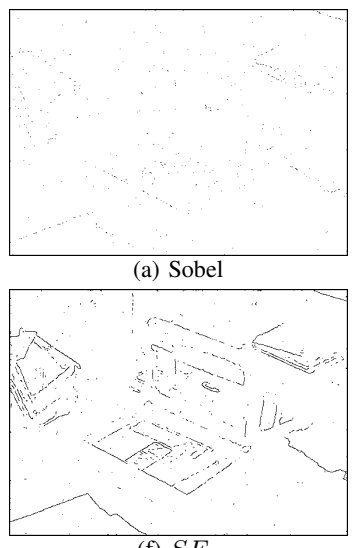

(f) $S F_{1}$

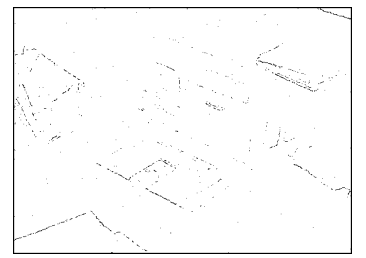

(b) Shen

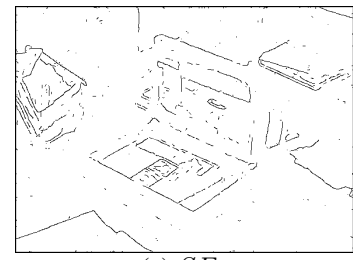

(g) $S F_{5}$

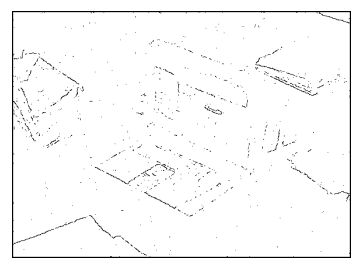

(c) Bourennane

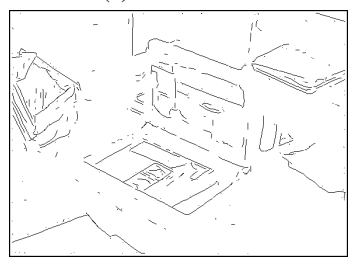

(h) AGK

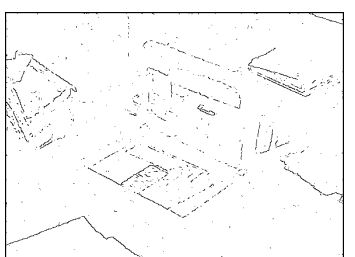

(d) Deriche

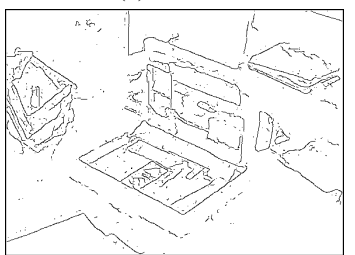

(i) $\mathrm{H}-\mathrm{K}$

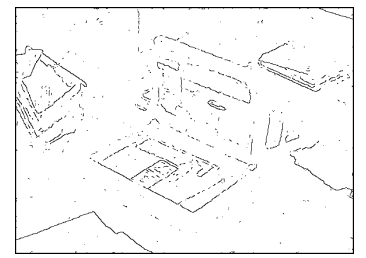

(e) Canny

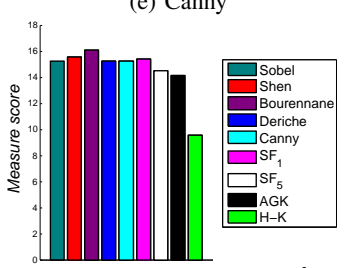

(j) Minimum scores of $\Delta^{k}$

Fig. 24. Comparison of the minimal score for the $\Delta^{k}$ measure concerning a real image and several edge detection methods.
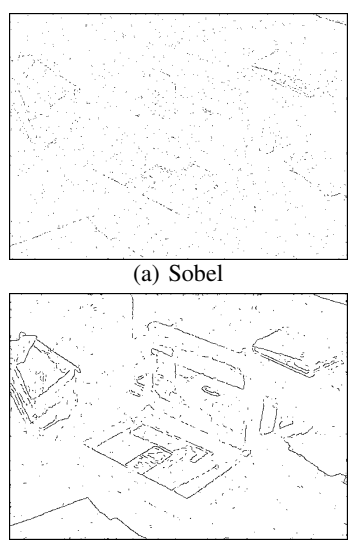

(f) $S F_{1}$

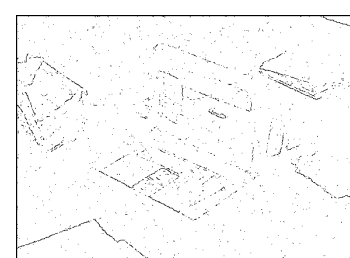

(b) Shen

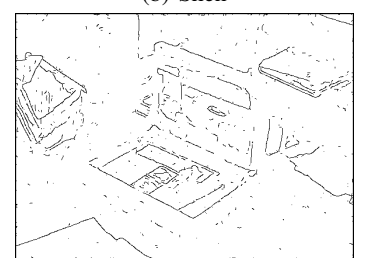

(g) $S F_{5}$

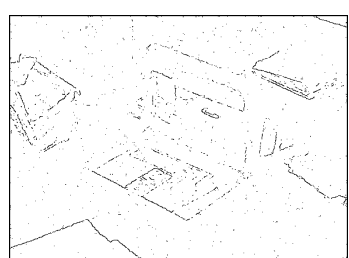

(c) Bourennane

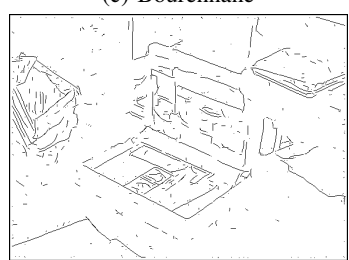

(h) AGK

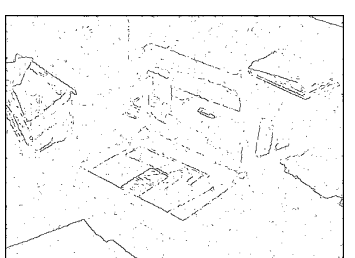

(d) Deriche

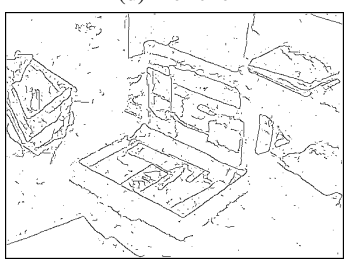

(i) $\mathrm{H}-\mathrm{K}$

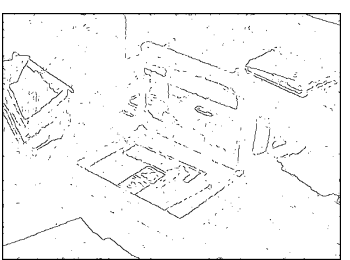

(e) Canny

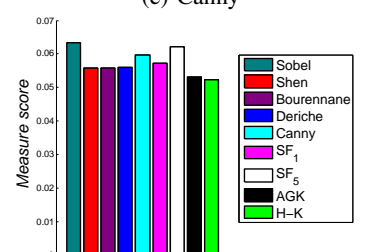

(j) Minimum scores of $\Psi$

Fig. 25. Comparison of the minimal score for the $\Psi$ measure concerning a real image and several edge detection methods.

latter compute a score depending mainly on the false positive and/or negative distances. By pointing out the drawbacks and advantages of each evaluation measure, in this work a new measure is proposed which takes into account the distance of all the misplaced pixels: false positives and false negatives. Moreover, one of the advantages of the developed method is a function which normalizes the evaluation. Indeed, the score is close to zero concerning good segmentation and increases to one concerning poor edge detection. The influence of the parameters of the new evaluation measure is detailed, theoretically and with concrete cases.
The rest of the paper is dedicated to the experimental results for synthetic and real data. Most of the edge detection evaluation methods are subjected to the following studies: addition of false negative points and/or false positive points, translation of the boundary, remoteness of false positive and false negative contour chains. Finally, as the minimum value of an edge detection evaluation corresponds to the threshold at which the edge detection is the best, the last experiment concerns the minimum value of the measures on image edges compared to the ground truth. This enables the performance of edge detection algorithms to be studied based on filtering 


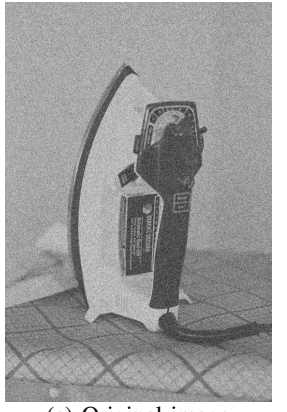

(a) Original image

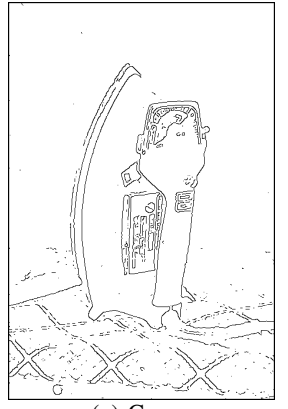

(g) Canny

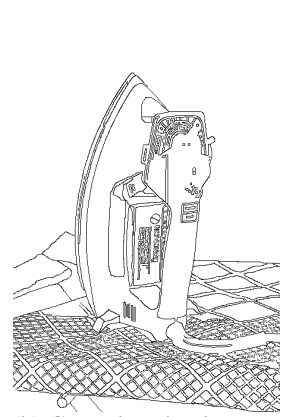

(b) Ground truth edge map

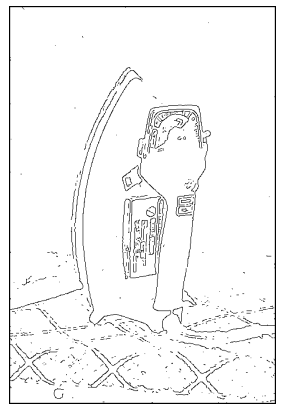

(h) $S F_{1}$
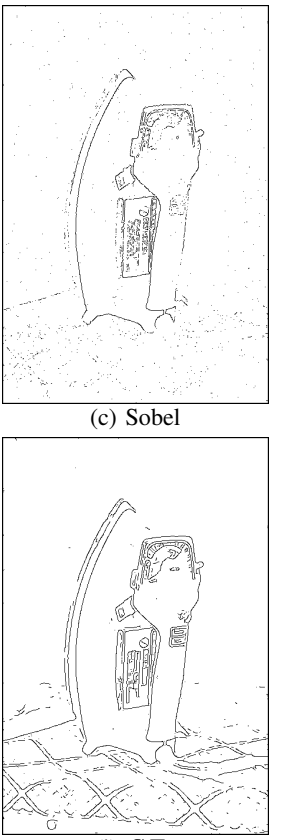

(i) $\mathrm{SF}_{5}$

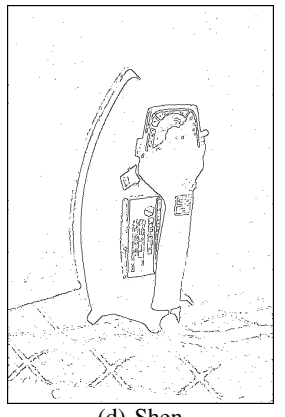

(d) Shen

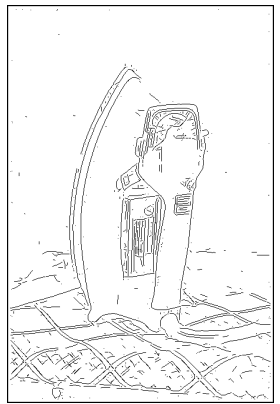

(j) AGK

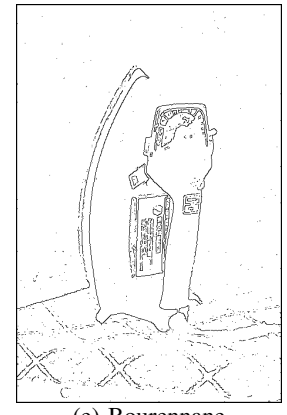

(e) Bourennane

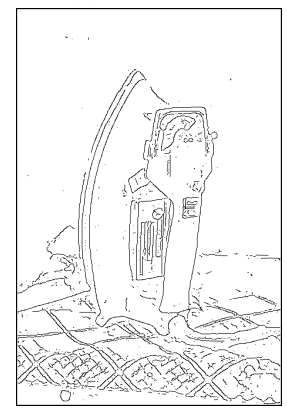

(k) $\mathrm{H}-\mathrm{K}$

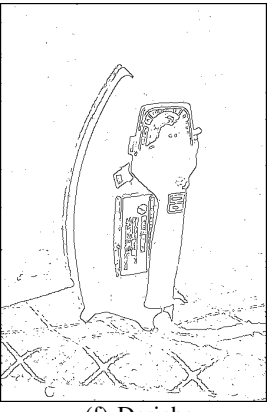

(f) Deriche

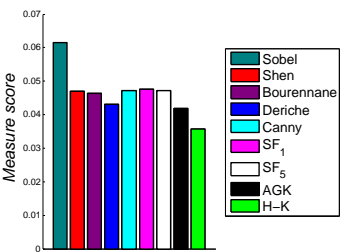

(l) Minimum scores of $\Psi$

Fig. 26. Comparison of the minimal score for the $\Psi$ measure concerning a real image and several edge detection methods.

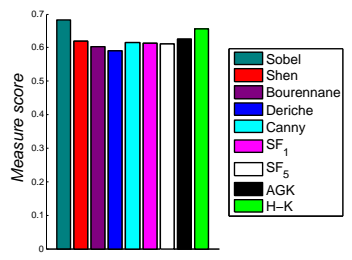

(a) $F_{\alpha}^{*}$

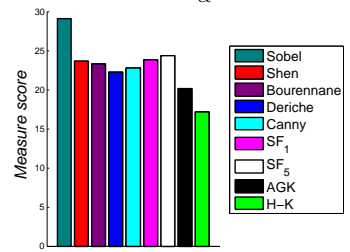

(f) $\mathrm{H}_{5 \%}$

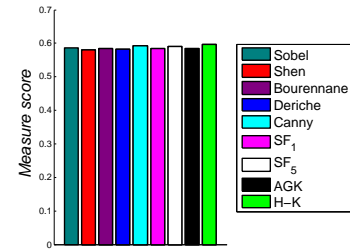

(k) $M F o M$

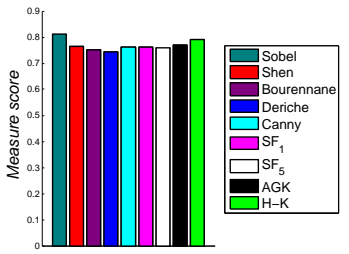

(b) $P_{m}^{*}$

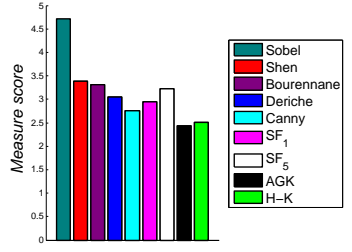

(g) $f_{2} d_{6}$

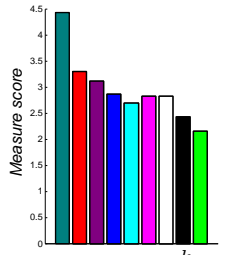

(1) $S_{k=1}^{k}$

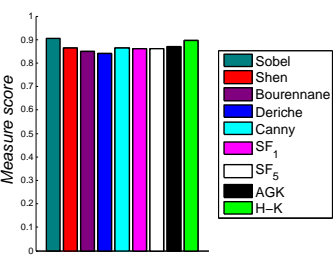

(c) $\chi_{2}^{*}$

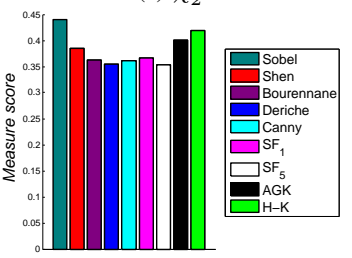

(h) $F o M$

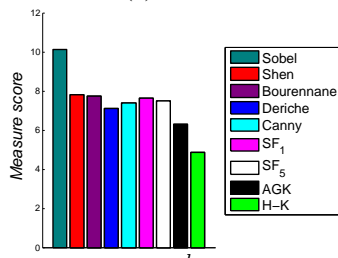

(m) $S_{k=1}^{k}$

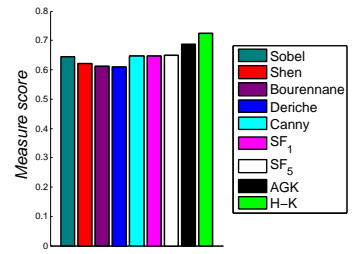

(d) $P h i *$

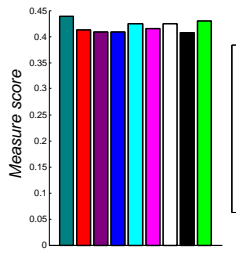

(i) $F$

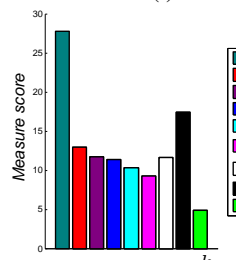

(n) $\Delta^{k}$

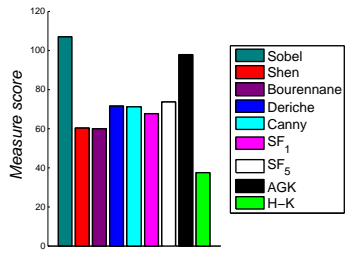

(e) $H$

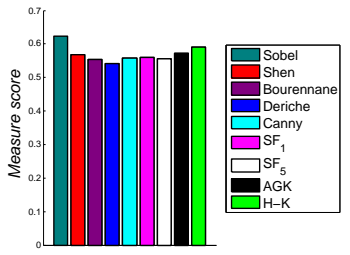

(j) $d_{4}$

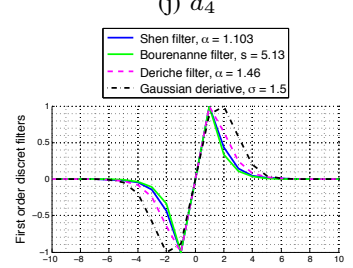

(o) Profile of the different filters

Fig. 27. Comparison of the minimal score for several edge evaluation measures concerning several edge detection methods of the image in Fig. 26 (a).

through the optimal threshold obtain by the supervised assessment. Therefore, as the proposed function of dissimilarity seems sufficiently robust to evaluate binary edge maps, we plan in a future study to compare more deeply several edge detection algorithms using the optimum threshold computed by the minimum of the evaluation.

\section{REFERENCES}

[1] I. E. Abdou and W. K. Pratt, Quantitative design and evaluation of enhancement/thresholding edge detectors, Proc. of the IEEE: Vol. 67(5), pp. 753-763, 1979.

[2] A. J. Baddeley, An error metric for binary images, Robust Comp. Vision: Quality of Vision Algorithms: pp. 59-78, 1992.
[3] M. Basseville, Distance measures for signal processing and pattern recognition, Signal Proc.: Vol. 18(4), pp. 349-369, 1989.

[4] E. Baudrier, F. Nicolier, G. Millon and S. Ruan, Binary-image comparison with local-dissimilarity quantification, Pattern Recognition: Vol. 41(5), pp. 1461-1478, 2008.

[5] I. A.G. Boaventura and A. Gonzaga, Method to evaluate the performance of edge detector, Brazilian Symposium on Comp. Graphics and Image Proc., 2009.

[6] E. Bourennane, P. Gouton, M. Paindavoine and F. Truchetet, Generalization of Canny-Deriche filter for detection of noisy exponential edge, Signal Proc.: Vol. 82(10), pp. 1317-1328, 2002.

[7] K. Bowyer, C. Kranenburg and S. Dougherty, Edge detector evaluation using empirical ROC curves, Comp. Vision and Image Understanding: pp. 77-103, 2001.

[8] J. Canny, A computational approach to edge detection, IEEE Trans. on Pattern Analysis and Machine Intel.: Vol. 6, pp. 679-698, 1986. 
[9] S. Chabrier, H. Laurent, C. Rosenberger, and B. Emile, Comparative study of contour detection evaluation criteria based on dissimilarity measures, EURASIP J. on Image and Video Proc.: Vol. 2008, pp. 1-13, 2008.

[10] D. Demigny, On optimal linear filtering for edge detection, IEEE Trans. on Image Proc., Vol.11(7), pp.728-737, 2002.

[11] R. Deriche, Using Canny's criteria to derive a recursively implemented optimal edge detector, Int. J. Computer Vision, Vol. 1, pp. 167-187, 1987.

[12] E. Deutsch and J. R. Fram, A quantitative study of the orientation bias of some edge detector schemes, IEEE Trans. on Computers: Vol. 27(3), pp. 205-213, 1978.

[13] M.P. Dubuisson, A.K. Jain, A modified Hausdorff distance for object matching, 12th IAPR Int. Conf. on Pattern Recognition: Vol. 1, pp. 566-568, 1994

[14] N. L. Fernández-Garcıa, R. Medina-Carnicer, A. and Carmona-Poyato, F. J. Madrid-Cuevas and M. Prieto-Villegas, Characterization of empirical discrepancy evaluation measures, Pattern Recognition Letters: Vol. 25(1), 35-47, 2003.

[15] W. T. Freeman and E. H. Adelson, The design and use of steerable filters, IEEE TPAMI: Vol. 13, pp. 89-906, 1991.

[16] J.M. Geusebroek, A. Smeulders, and J. van de Weijer, Fast anisotropic gauss filtering, ECCV 2002, pp. 99-112, 2002.

[17] J. Gimenez, J. Martinez and A. G. Flesia, Unsupervised edge map scoring: A statistical complexity approach, Comp. Vision and Image Understanding: Vol. 122, pp. 131-142, 2014.

[18] A. B. Goumeidane, M. Khamadja, B., Belaroussi, H., Benoit-Cattin and C. Odet, New discrepancy measures for segmentation evaluation, IEEE ICIP: Vol. 2, pp. 411-414, 2003.

[19] C. Grigorescu, N. Petkov and M.A. Westenberg, Contour detection based on nonclassical receptive field inhibition, IEEE Trans. on Image Proc.: Vol. 12(7), pp. 729-739, 2003.

[20] R. M. Haralick, Digital step edges from zero crossing of second directional derivatives, IEEE Trans. on Pattern Analysis and Machine Intel.: Vol. 6(1), pp. 58-68, 1984.

[21] M. D. Heath, S. Sarkar, T. Sanocki, and K. W. Bowyer, A robust visual method for assessing the relative performance of edge-detection algorithms, IEEE Trans. on Pattern Analysis and Machine Intel.: Vol. 19(12), pp. 1338-1359, 1997.

[22] B. Hemery, H. Laurent, B. Emile, and C. Rosenberger, Comparative study of localization metrics for the evaluation of image interpretation systems, J. of Elec. Imaging: Vol. 19(2), pp. 023017-023017, 2010

[23] X. Hou, A. Yuille and C. Koch, Boundary detection benchmarking: Beyond f-measures, IEEE Conference on Comp. Vision and Pattern Recognition, pp. 2123-2130, 2013.

[24] D. P. Huttenlocher and W. J. Rucklidge, A multi-resolution technique for comparing images using the Hausdorff distance, IEEE Trans. on Pattern Analysis and Machine Intel.: Vol. 15(9), pp. 850-863, 1993.

[25] M. Jacob and M. Unser, Design of steerable filters for feature detection using canny-like criteria, IEEE TPAMI, Vol. 26(8), pp. 1007-1019, 2004.

[26] R. K. Falah, P. W. Bolon, J. P. Cocquerez, A Region-Region and RegionEdge Cooperative Approach of Image Segmentation, IEEE Int. Conf. on Image Proc.: Vol. 3, pp. 470-474, 1994.

[27] U. Köthe, Reliable low-level image analysis, Habilitation thesis, 2007.

[28] O. Laligant, F. Truchetet, F. Meriaudeau, Regularization preserving localization of close edges, IEEE Signal Proc. Letters, Vol. 14(3), 185-188, 2007.

[29] S. U. Lee, S. Y. Chung, and R. H. Park, A comparative performance study of several global thresholding techniques for segmentation, CVGIP, Vol. 52(2), pp. 171-190, 1990.

[30] C. Lopez-Molina, H. Bustince, J. Fernandez, P. Couto and B. De Baets, A gravitational approach to edge detection based on triangular norms, Pattern Recognition: Vol. 43(11), pp. 3730-3741, 2010.

[31] C. Lopez-Molina, B. De Baets and H. Bustince, Quantitative error measures for edge detection, Pattern Recognition: Vol. 46(4), pp. 11251139,2013

[32] C. Lopez-Molina, M. Galar, H. Bustince, and B. De Baets, On the impact of anisotropic diffusion on edge detection, Pattern Recognition: Vol. 47 (1), pp. 270-281, 2014.

[33] B. Magnier, F., Comby, O., Strauss, J., Triboulet and C. Demonceaux Highly Specific Pose Estimation with a Catadioptric Omnidirectional Camera, IEEE Int. Conf. on Imaging Systems and Techniques, pp. 229-233, 2010.

[34] B. Magnier, P. Montesinos, and D. Diep, Texture Removal by Pixel Classification using a Rotating Filter, IEEE Int. Conf. on Acoustics, Speech, and Signal Proc., pp. 1097-1100, 2011.
[35] B. Magnier, P. Montesinos and D. Diep, Fast anisotropic edge detection using gamma correction in color images, IEEE Int. Symposium on Image and Signal Proc. and Analysis, pp. 212-217, 2011.

[36] B. Magnier, A. Aberkane, P. Borianne, P. Montesinos, and C. Jourdan, Multi-scale crest line extraction based on half gaussian kernels, IEEE Int. Conf. on Acoustics, Speech, and Signal Proc., pp. 5105-5109, 2014.

[37] B. Magnier, A. Le and A. Zogo, A quantitative error measure for the evaluation of roof edge detectors, IEEE Int. Conf. on Imaging Systems and Techniques, pp. 429-434, 2016.

[38] D. Marr and E. Hildreth, Theory of Edge Detection, Proceedings of the Royal Society of London. Series B, Biological Sciences: Vol. 207 (1167), pp. 187-217, 1980.

[39] D. R. Martin, C. C. Fowlkes and J. Malik, Learning to detect natural image boundaries using local brightness, color, and texture cues, IEEE Trans. on Pattern Analysis and Machine Intel.: Vol. 26(5), pp. 530-549, 2004.

[40] C. Odet, B. Belaroussi and H. Benoit-Cattin, Scalable discrepancy measures for segmentation evaluation, IEEE Int. Conf. on Image Proc.: Vol. 1, pp. 785-788, 2002.

[41] N. Otsu, A threshold selection method from gray-level histograms, IEEE Trans. on Systems Man and Cybernetics: Vol. 9, pp. 62-66, 1979.

[42] K. Panetta, C.Gao, S.Agaian,and S.Nercessian, Non-reference medical image edge map measure, J. of Bio. Imaging: Vol. 2014, pp. 1-12, 2014.

[43] K. Panetta, C. Gao, S. Agaian and S. Nercessian, A New ReferenceBased Edge Map Quality Measure, IEEE Trans. on Systems Man and Cybernetics: Systems: Vol. 46(11), pp. 1505-1517, 2016.

[44] G. Papari and N.Petkov, Edge and line oriented contour detection: State of the art, Image and Vision Computing: Vol. 29(2) , pp. 79-103, 2011.

[45] J. Paumard, Robust comparison of binary images, Pattern Recognition Letters: Vol. 18(10), pp. 1057-1063, 1997.

[46] T. Peli and D. Malah, A study of edge detection algorithms, CGIP: Vol. 20(1), pp. 1-21, 1982.

[47] A. J. Pinho and L. B.Almeida, Edge detection filters based on artificial neural networks. In Int. Conf. on Image Analysis and Proc., Springer Berlin Heidelberg, pp. 159-164, 1995.

[48] R. Román-Roldán, J. F. Gómez-Lopera, C. Atae-Allah, J. Martı'nezAroza and P.L. Luque-Escamilla, A measure of quality for evaluating methods of segmentation and edge detection, Pattern Recognition: Vol. 34(5), 969-980, 2001.

[49] A. Rosenfeld and M. Thurston, Edge and curve detection for visual scene analysis, IEEE Trans. on Comp.: Vol. 100(5), pp. 562-569, 1971.

[50] J. Shen and S. Castan, An optimal linear operator for edge detection, IEEE CVPR, Vol. 86, pp. 109-114, 1986.

[51] P. Sneath and R. Sokal, Numerical taxonomy. The principles and practice of numerical classification, 1973.

[52] K. C. Strasters and J. J. Gerbrands, Three-dimensional image segmentation using a split, merge and group approach, Pattern Recognition Letters: Vol. 12(5), pp. 307-325, 1991.

[53] I.E. Sobel, Camera Models and Machine Perception, PhD Thesis, Stanford University, 1970.

[54] V. Torre and T.A. Poggio, On edge detection, IEEE Trans. on Pattern Analysis and Machine Intel.: Vol. 8, (2), pp. 147-163, 1986.

[55] R. Usamentiaga, D. F. García, C. López, and D. González, A method for assessment of segmentation success considering uncertainty in the edge positions, EURASIP J. on Applied Signal Proc., Vol. 2006, pp. 207-207, 2006

[56] S. Venkatesh and P.L. Rosin, Dynamic threshold determination by local and global edge evaluation, Comp. Vision, Graphics, and Image Proc.: Vol. 57(2), pp. 146-160, 1995.

[57] Z. Wang, , A.C. Bovik, H.R. Sheikh and E.P. Simoncelli, Image Quality Assessment: from Error Visibility to Structural Similarity, IEEE Trans. on Pattern Analysis and Machine Intel.: Vol. 13(4), pp. 600-612, 2004.

[58] D. L. Wilson, A. J. Baddeley and R. A. Owens, A new metric for greyscale image comparison, Int. J. of Comp. Vision: Vol. 24(1), 5-17, 1997.

[59] W.A. Yasnoff, W. Galbraith and J.W. Bacus, Error measures for $o b$ jective assessment of scene segmentation algorithms, Analytical and Quantitative Cytology: Vol. 1(2), pp. 107-121, 1978.

[60] Y. Yitzhaky and E. Peli, A method for objective edge detection evaluation and detector parameter selection, IEEE Trans. on Pattern Analysis and Machine Intel., vol. 25, no. 8, pp. 10271033, 2003.

[61] C. Zhao, W. Shi and Y. Deng, A new Hausdorff distance for image matching, Pattern Recognition Letters: Vol. 26(5), pp. 581-586, 2005. 